\title{
Classes of Operations in Quantum Theory
}

\author{
C. M. EDWARDS \\ The Queen's College, Oxford \\ Received April 24, 1970
}

\begin{abstract}
Recent work of Davies and Lewis has shown how partially ordered vector spaces provide a setting in which the operational approach to statistical physical systems may be studied. In this paper, certain physically relevant classes of operations are identified in the abstract framework, some of their properties are derived and applications to the Von Neumann algebra model for quantum theory are discussed.
\end{abstract}

\section{§1. Introduction}

In a previous paper [7], the operational approach to the theory of statistical physical systems, originally suggested by Haag and Kastler [13] and recently formulated in terms of partially ordered vector spaces by Davies and Lewis [4], was discussed in some detail.

Briefly, the abstract formulation may be described as follows. Regarding states as (equivalence classes of) statistical ensembles of the physical system under examination, the set of states may be represented by a generating cone $K$ for a real vector space $V$, where addition of elements of $K$ represents a process in which the corresponding states are mixed and where multiplication by positive scalars represents the proportional increase in the number of copies of the system in the corresponding state. The element 0 of $K$ represents the state in which there are no systems. A state $f$ may be thought of as a beam of systems emerging from some conditioning apparatus at a constant rate $e(f)$. Then, $e$ extends to a strictly positive linear functional, called the strength functional, on $V$ and the set $B$ of states $f$ such that $e(f)=1$ forms a base for the cone $K . B$ is said to be the set of normalized states of the system. The Minkowski functional on the convex hull of $B \cup(-B)$ defines a semi-norm on $V$ which coincides with $e$ on $K$. The assumption that countable mixtures of states may be formed leads to the conclusion that this semi-norm is, in fact, a norm with respect to which $V$ is complete [8]. In general, $K$ need not be closed for the norm topology although a result of Ellis [11] shows that if $\bar{K}, \bar{B}$ are the completions of $K, B$ respectively, then $\bar{K}$ is a cone in $V$ with base $\bar{B}$ and that the associated 
semi-norm coincides with the norm on $V$. Therefore, there is little loss of generality in supposing that $K$ is closed in $V$. An operation on the system may be regarded as some kind of filtering process on the states and may be described by an affine mapping $j$ from $K$ to itself satisfying $j(0)=0, e(j(f)) \leqq e(f), \forall f \in K$. Each such mapping has a unique extension to a positive, norm non-increasing linear operator on $V$ and hence, the set $\mathscr{P}$ of operations may be identified with the positive part of the unit ball in the space $\mathfrak{E}(V)$ of bounded linear operators on $V$. The dual space $V^{*}$ of $V$ possesses a dual cone $K^{*}$ containing $e$ as an order unit and the ordering of $V^{*}$ by $K^{*}$ is Archimedean. Associated with each operation $j$, there is an element $T(j) \in V^{*}$ such that $0 \leqq T(j) \leqq e$, defined by $T(j)(f)=e(j(f)), \forall f \in K . T(j)$ is said to be the simple observable corresponding to the operation $j$ and the order interval $[0, e]=2$ is said to be the set of simple observables of the system. It is easily verified that the mapping $j \mapsto T(j)$ sends $\mathscr{P}$ onto 2 . For $f \in K, T(j)(f) / e(f)$ is the transmission probability for the state $f$ under the operation $j$. It follows that, if only experiments which count systems are allowed, $\mathscr{2}$ forms the set of all measurable quantities. A restriction of the physical system may be regarded physically as some process which reduces the number of states of the system in a particular way. The set of states of the restricted system forms a subset $H$ of $K$. Simple physical considerations lead to the conclusion that $H$ is an extremal set of $K$ satisfying a certain extension property for bounded positive affine functionals on $H$. The former stems from the obvious properties of mixed states whilst the latter is suggested by the requirement that the set of simple observables of the restricted system is precisely the set of simple observables of the whole system where two are identified when they have equal probabilities on the set of restricted states. The possibility that complementary restrictions exist suggests that the annihilator $H^{0}$ of $H$ in $V^{*}$ be positively generated and the Heisenberg-Schrödinger duality between sets of simple observables and sets of states suggests that $H$ is norm closed in $K$.

An example of the situation described above is obtained by choosing $K$ to be the set of positive normal linear functionals on a Von Neumann algebra $\mathfrak{B}$ acting on a Hilbert space $X$ and having identity $e$. Then, $V$ is the space of hermitean elements in the pre-dual $\mathfrak{B}_{*}$ of $\mathfrak{B}, B$ is the set of normal states of $\mathfrak{B}, V^{*}$ is the space of self-adjoint elements of $\mathfrak{B}$ and $K^{*}$ is the set of positive elements of $\mathfrak{B}$. The set $\mathscr{Q}$ of simple observables in this case reduces to the set of positive operators $A$ in $\mathfrak{B}$ such that $A \leqq e .2$ is an ultraweakly compact convex subset of $V^{*}$ and by the KreinMilman theorem is the ultraweak closure of the convex hull of its set $E(2)$ of extreme points. $E(2)$ is the set of projections in $\mathfrak{B}[16]$. A particular case of this example is obtained by choosing $\mathfrak{B}$ to be the Von Neumann envelope of an abstract $C^{*}$-algebra $\mathfrak{I}$. This leads to the approach 
to quantum theory suggested by Segal [19], Haag and Kastler [13]. The set of restrictions in the Von Neumann algebra model is in one-one correspondence with the set of central projections in $\mathfrak{B}$. In fact, for each central projection $E$ in $\mathfrak{B}$, the set of states of the corresponding restricted system is the set of positive normal linear functionals on the Von Neumann algebra $E \mathfrak{B} E$ acting on $E X$, whilst the set of simple observables of the restricted system is the set of positive elements $A$ of $E \mathfrak{B} E$ such that $A \leqq E$. In the particular case in which $\mathfrak{B}$ is the Von Neumann envelope of the $C^{*}$-algebra $\mathfrak{A}$, the set of restrictions is also in one-one correspondence with the set of quasi-equivalence classes of representations of $\mathfrak{U}$.

The main object of this paper is to investigate the properties of various physically relevant subsets of $\mathscr{Q}$ and $\mathscr{P}$ in the abstract formulation and attempt to identify them in the Von Neumann algebra model. It should be remarked that $\mathscr{P}$ is usually an extremely large set even in the simplest examples and it would, therefore, be unreasonable to expect that a characterization of all elements of $\mathscr{P}$ to be possible [20]. In $\S 3$, the structure of $\mathscr{Q}$ and then of $\mathscr{P}$ is examined. It is shown that the set $E(2)$ of extreme points of 2 plays quite an important role in measurement theory (Prop. 3.1) and it is shown that the set of operations, which satisfy conditions similar to those imposed by Von Neumann [21] in his measurement theory, lead to simple observables in $E(2)$ (Prop. 3.6). A still more restrictive class of operations is defined and it is shown that the effect of such operations is to produce sets of states of restrictions of the system (Prop. 3.12). In this connection, Prop. 3.11 owes much to Alfsen [1] and Gerzon [12]. Several of the classes of operations defined in $\S 3$ stem from the work of Davies and Lewis [4].

In $\S 4$, the results of $\S 3$ are applied to the Von Neumann algebra model for quantum mechanics. It is shown that the various classes of operations can be described by means of projections in the Von Neumann algebra. In $\S 5$, application is made to two particular classes of operations which are important in algebraic quantum theory but which have not been discussed in the abstract framework. It is not claimed that the results in $\S \$, 5$ are the strongest possible, for the Von Neumann algebra $\mathfrak{B}$ considered is arbitrary. Clearly, more powerful results may be obtained by choosing $\mathfrak{B}$ to be a Von Neumann algebra or factor of fixed type.

\section{$\S 2$. Preliminaries}

For the most part the definitions and notation of [7] are maintained. A non-empty subset $K$ of a real vector space $V$ is said to be a cone if $K+K \subset K, \alpha K \subset K, \alpha \geqq 0$ and $K \cap(-K)=\{0\}$. The cone defines a partial ordering on elements of $V$ if $f \geqq g$ is defined to mean that $f-g \in K$. If 
$K-K=V, K$ is said to be a generating cone. A subset $H$ of $K$ is said to be an extremal set (support) of $K$ if $H+H \subset H, \alpha H \subset H, \forall \alpha \geqq 0$ and if $f \in H, f=t f_{1}+(1-t) f_{2}, f_{1}, f_{2} \in K, t \in(0,1)$ implies $f_{1}, f_{2} \in H$. The union $H^{\prime}$ of all extremal sets $G$ in $K$ such that $H \cap G=\{0\}$ is said to be the complementary set of $H$. If $H^{\prime}$ is an extremal set and every element $f$ of $K$ has a unique decomposition $f=g+h, g \in H, h \in H^{\prime}$, following Alfsen and Anderson [1], $H$ is said to be a split extremal set. A nonempty convex subset $B$ of $K$ is said to be a base for $K$ if, for each $f \in K$, $f \neq 0$, there exist uniquely $g \in B, \alpha>0$ such that $f=\alpha g$. A linear functional $e$ on $V$ is said to be strictly positive if $e(f) \geqq 0, \forall f \in K$ and $f \in K$, $e(f)=0$ implies $f=0 . K$ possesses a base $B$ if and only if there exists a strictly positive linear functional $e$ on $V$ and in this case $B$ may be written as $\{f: f \in K, e(f)=1\}$. If, for $f \in V$,

$$
\|f\|_{B}=\inf \{\lambda \geqq 0: f \in \lambda \operatorname{conv}(B \cup(-B))\}
$$

where $\operatorname{conv}(B \cup(-B))$ is the convex hull of $B \cup(-B)$, then, providing that $K$ generates $V,\|\cdot\|_{B}$ is a semi-norm on $V$ and

$$
\|f\|_{B}=\inf \left\{e\left(f_{1}\right)+e\left(f_{2}\right): f=f_{1}-f_{2}, f_{1}, f_{2} \in K\right\} .
$$

$\|\cdot\|_{B}$ is a norm on $V$ if and only if $\operatorname{conv}(B \cup(-B))$ is linearly bounded in which case $(V, B)$ is said to be a base norm space [10].

If $S$ is any convex subset of a real vector space, a non-empty convex subset $F$ of $S$ is said to be a face of $S$ if, $f \in F, f=t f_{1}+(1-t) f_{2} f_{1}, f_{2}$ $\in S, t \in(0,1)$ implies $f_{1}, f_{2} \in F$. If $F$ is a face of $S$, the union $F^{\prime}$ of all faces of $S$ disjoint from $F$ is said to be the complementary set of $F$. If $F^{\prime}$ is a face and every $f \in S$ has a unique decomposition $f=t g+(1-t) h, g \in F$, $h \in F^{\prime}, t \in[0,1], F$ is said to be a split face [1]. If $\{f\} \subset S$ is a face of $S, f$ is said to be an extreme point of $S$ and the set of extreme points of $S$ is denoted by $E(S)$.

Let $(V, B)$ be a complete base norm space with norm closed cone $K$. Then, there exists a bijection $H \mapsto F$ between the set of extremal sets $H$ of $K$ and the set of faces $F$ of $B$ defined by $F=H \cap B, H=\{\alpha f: \alpha \geqq 0, f \in F\}$. $H$ is norm closed in $K$ if and only if $F$ is norm closed in $B$. Let $V^{*}$ be the dual of $V$ and let $K^{*}=\left\{T: T \in V^{*}, T(f) \geqq 0, \forall f \in K\right\}$. Then $K^{*}$ is a $\sigma\left(V^{*}, V\right)$ closed generating cone for $V^{*}$ and if $B=\{f: f \in K, e(f)=1\}$, $e \in K^{*}$ and for $T \in V$, there exists $\lambda \geqq 0$ such that $-\lambda e \leqq T \leqq \lambda e . K^{*}$ is said to be the cone dual to $K$ and $e$ is said to be an order unit for the ordering of $V^{*}$ by $K^{*}$. In fact, if $T \in V^{*}, T \leqq \alpha T^{\prime}$, some $T^{\prime} \in K^{*}, \forall \alpha>0$, $T \leqq 0$ and in this case the ordering of $V^{*}$ by $K^{*}$ is said to be Archimedean. Moreover, if for $T \in V^{*}$,

$$
\|T\|_{e}=\inf \{\lambda \geqq 0,-\lambda e \leqq T \leqq \lambda e\},
$$


$\|\cdot\|_{e}$ is a norm on $V^{*}$ and $\left(V^{*}, e\right)$ is said to be an Archimedean ordered order unit space. In fact the norm $\|.\|_{e}$ coincides with the Banach space norm and the order interval $[-e, e]$ is the unit ball in $\left(V^{*}, e\right)$. The cones $K, K^{*}$ are compatible in the sense that $K=\left\{f: f \in V, T(f) \geqq 0, \forall T \in K^{*}\right\}$.

For each subset $L$ of $V$ or $J$ of $V^{*}$, let $L^{0}, J_{0}$ be the annihilators of $L$ in $V^{*}$ and $J$ in $V$ respectively. $L^{0}, J_{0}$ are subspaces which are $\sigma\left(V^{*}, V\right)$ and norm closed respectively. If $L \subset L \cap K-L \cap K$, in which case $L$ is said to be positively generated, $L^{0} \cap K^{*}$ is an extremal set in $K^{*}$ and if $J$ is positively generated, $J_{0} \cap K$ is an extremal set in $K$.

If $S, S^{\prime}$ are convex subsets of real vector spaces a mapping $\phi: S \rightarrow S^{\prime}$ is said to be affine if and only if $\phi(t f+(1-t) g)=t \phi(f)+(1-t) \phi(g)$, $\forall f, g \in S, t \in[0,1]$. When $\phi$ maps $S$ one-one onto $S^{\prime}, \phi$ is said to be an affine isomorphism. When $S^{\prime}$ is a convex subset of the real line $\boldsymbol{R}$, an affine mapping is said to be an affine functional. If $K, K^{\prime}$ are cones in real vector spaces a mapping $\phi: K \rightarrow K^{\prime}$ is said to be affine if $\phi(f+g)$ $=\phi(f)+\phi(g), \phi(\alpha f)=\alpha \phi(f), \forall f, g \in K, \alpha \geqq 0$, and if $K^{\prime} \subset \boldsymbol{R}, \phi$ is said to be an affine functional. If $(V, B)$ is a complete base norm space with norm closed cone $K, V^{*}$ may be identified with the space of all bounded affine functionals on $B$ or $K$ and for $T \in V^{*}$,

$$
\|T\|_{e}=\sup \{|T(f)|: f \in B\}=\sup \{|T(f)| / e(f): f \in K\} .
$$

A Borel space $(\mathscr{S}, \mathscr{B})$ is a space $\mathscr{S}$ together with a set $\mathscr{B}$ of subsets of $\mathscr{S}$ closed under the formation of countable unions, countable intersections and complements and contains $\mathscr{S}$ as an element. If $V$ is a real topological space and $K$ is a cone in $V$, a mapping $\mu: \mathscr{B} \rightarrow K$ such that for any family $\left\{M_{n}\right\}$ of mutually disjoint elements of $\mathscr{B}$,

$$
\mu\left(\bigcup_{n=1}^{\infty} M_{n}\right)=\sum_{n=1}^{\infty} \mu\left(M_{n}\right)
$$

where the sum converges in the topology of $V$, is said to be a $K$-valued measure on $(\mathscr{S}, \mathscr{B})$. If the range of $\mu$ lies in some subset $K^{\prime}$ of $K, \mu$ is said to be a $K^{\prime}$-valued measure.

\section{§ 3. Operations and Simple Observables}

Using [4], the following list of postulates were proposed in [7] for the description of statistical physical systems.

1. The set of states of the system is represented by a norm closed cone $K$ in a complete base norm space $(V, B)$.

2. The set of operations on the system is represented by the set $\mathscr{P}$ of positive elements in the unit ball in the space $\mathfrak{S}(V)$ of bounded linear operators on $V$. 
3. The set of simple observables of the system is represented by the set 2 of elements $A$ in the dual space $\left(V^{*}, e\right)$ of $(V, B)$ satisfying $0 \leqq A \leqq e$ where the ordering is defined by the dual cone $K^{*}$ of $K$.

4. The set of instruments on the system is represented by the set of $\mathscr{P}$-valued measures $\mathscr{E}$ on Borel spaces $(\mathscr{S}, \mathscr{B})$ such that $e(\mathscr{E}(\mathscr{S}) f)=e(f)$, $\forall f \in K$.

5. The set of observables of the system is represented by the set of 2-valued measures $\mathscr{A}$ on Borel spaces $(\mathscr{S}, \mathscr{B})$ such that $\mathscr{A}(\mathscr{S})=e$.

6. The set of states of a restriction of the system is represented by a norm closed extremal set $H$ of $K$ satisfying,

(i) If $L=H-H, F=H \cap B,(L, F)$ is a complete base norm space with norm closed generating cone $H$.

(ii) If $\tilde{A}$ is an affine functional on $H$ such that $0 \leqq \tilde{A}(f) \leqq e(f), \forall f \in H$, there exists an affine functional $A$ on $K$ such that $0 \leqq A(f) \leqq e(f), \forall f \in K$ and $A(f)=\tilde{A}(f), \forall f \in H$.

(iii) $H^{0}$ is positively generated.

The first part of this section is devoted to a discussion of the general properties of $\mathscr{P}$ and $\mathscr{Q}$ and the mapping $j \mapsto T(j)$ from $\mathscr{P}$ onto $\mathscr{Q}$ defined by

$$
T(j)(f)=e(j(f)), \quad \forall f \in K .
$$

2 is the intersection of the $\sigma\left(V^{*}, V\right)$ compact unit ball in $\left(V^{*}, e\right)$ with the $\sigma\left(V^{*}, V\right)$ closed cone $K^{*}$ in $V^{*}$ and is therefore $\sigma\left(V^{*}, V\right)$ compact. Let $E(2)$ be the set of extreme points of $\mathscr{Q}$. Elements of $E(\mathscr{Q})$ are said to be extreme simple observables. Clearly, $0, e \in E(\mathscr{Q}), A \in E(\mathscr{Q}), A \neq 0$ implies that $\|A\|=1$ and $A \in E(2)$ if and only if $e-A \in E(\mathscr{2})$. The Krein-Milman theorem shows that for $A \in \mathscr{Q}, f \in K, \varepsilon>0$, there exist $\left\{A_{i}\right\} \subset E(\mathscr{Q})$, $i=1,2, \ldots n,\left\{t_{i}\right\} \subset[0,1], i=1,2, \ldots n$ such that

$$
\begin{gathered}
\sum_{i=1}^{n} t_{i}=1, \\
\left|A(f)-\sum_{i=1}^{n} t_{i} A_{i}(f)\right|<\varepsilon .
\end{gathered}
$$

Let $\boldsymbol{Z}$ denote the set of integers and for $i \in \boldsymbol{Z}$, define

$$
\begin{aligned}
\mathscr{A}_{f, \varepsilon}(i) & =t_{i} A_{i}, \quad 0<i \leqq n, \\
& =0, \quad i=0, i<-n, i>n, \\
& =t_{-i}\left(e-A_{-i}\right),-n \leqq i<0 .
\end{aligned}
$$


For $\Lambda \subset \boldsymbol{Z}$, let $\Lambda_{+}=\{i: i \in \Lambda, i \geqq 0\}, \Lambda_{-}=\{i: i \in \Lambda, i<0\}$ and define

$$
\begin{aligned}
\mathscr{A}_{f, \varepsilon}(\Lambda) & =\sum_{i \in A} \mathscr{A}_{f, \varepsilon}(i) \\
& =\sum_{i \in A_{+}} t_{i} A_{i}+\sum_{i \in A_{-}} t_{i}\left(e-A_{i}\right) \\
& \leqq \sum_{i=1}^{n} t_{i} A_{i}+\sum_{i=1}^{n} t_{i}\left(e-A_{i}\right) \\
& =e .
\end{aligned}
$$

Hence, $\mathscr{A}_{f, \varepsilon}(\Lambda) \in \mathscr{Q}$ and if $\left\{\Lambda_{m}\right\}$ is a countable family of disjoint subsets of $\boldsymbol{Z}$,

$$
\mathscr{A}_{f, \varepsilon}\left(\bigcup_{m=1}^{\infty} \Lambda_{m}\right)=\sum_{m=1}^{\infty} A_{f, \varepsilon}\left(\Lambda_{m}\right) \text {. }
$$

Further, $\mathscr{A}_{f, \varepsilon}(\boldsymbol{Z})=e$ and hence $\mathscr{A}_{f, \varepsilon}$ is an observable defined on $\boldsymbol{Z}$. Finally,

$$
\mathscr{A}_{f, \varepsilon}\left(\boldsymbol{Z}_{+}\right)=\sum_{i=1}^{n} t_{i} A_{i}, \mathscr{A}_{f, \varepsilon}\left(\boldsymbol{Z}_{-}\right)=e-\sum_{i=1}^{n} t_{i} A_{i} .
$$

Following [4], an observable is said to be discrete if it is defined on a discrete space and finite if it is defined on a finite space. A discrete observable $\mathscr{A}$ based on $\mathscr{S}$ is said to be extreme if $\mathscr{A}(s) \in E(\mathscr{2}), \forall s \in \mathscr{S}$. A restatement of the Krein-Milman theorem in these terms follows from the discussion above.

Proposition 3.1. For each $A \in \mathscr{Q}, f \in K, \varepsilon>0$, there exists a finite extreme observable $\mathscr{A}_{f, \varepsilon}$ defined on a subset $\mathscr{S}$ of $\boldsymbol{Z}$ such that

$$
\left|A(f)-\mathscr{A}_{f, \varepsilon}\left(\mathscr{S}_{+}\right)\right|<\varepsilon, \quad\left|(e-A)(f)-\mathscr{A}_{f, \varepsilon}\left(\mathscr{S}_{-}\right)\right|<\varepsilon .
$$

It follows that the set $E(2)$ fulfils quite an important role in measurement theory since every simple observable can be approximated by finite extreme observables.

Let $\mathscr{K}=\{j: j \in \mathfrak{L}(V), j(K) \subset K\}$. Then, $\mathscr{K}$ is a strongly closed cone in $\mathfrak{L}(V)$, not, in general, generating [11], and $\mathscr{P}$ is the intersection of $\mathscr{K}$ with the unit ball in $\mathscr{L}(V)$. It follows that $\mathscr{P}$ is a uniformly closed convex set in $\mathfrak{L}(V)$. Let $\mathscr{P}^{*}$ denote the set of all $\sigma\left(V^{*}, V\right)$ continuous linear mappings from $V^{*}$ to itself which are norm non-increasing and map $K^{*}$ into $K^{*}$. Then, if $j^{*}$ denotes the adjoint of $j \in \mathscr{P}$, it is clear that $j^{*} \in \mathscr{P}^{*}$ and that the mapping $j \mapsto j^{*}$ is an affine isomorphism between $\mathscr{P}$ and $\mathscr{P}^{*}$. It follows that any study of $\mathscr{P}$ is automatically bound up with a study of $\mathscr{P} *$.

For $j \in \mathscr{P}, T(j) \in \mathscr{Q}$ is defined by $T(j)(f)=e(j(f)), \forall f \in K$, and it is clear that $j \mapsto T(j)$ is an affine mapping from $\mathscr{P}$ into $\mathscr{Q}$. Further, for $A \in \mathscr{Q}$, 
if $j \in \mathscr{P}$ is defined by

$$
j(f)=A(f) g, \quad g \in B, \forall f \in K,
$$

then $T(j)=A$ and hence the mapping $j \mapsto T(j)$ sends $\mathscr{P}$ onto 2 . For $A \in \mathscr{Q}, T^{-1}(A)$ is said to be an isotony class of operations corresponding to the the simple observable $A$, and two operations in the same isotony class are said to be isotonic.

Notice that, for $j \in \mathscr{P}$,

$$
\begin{aligned}
\|j\| & =\sup \left\{\|j(f)\|_{B} /\|f\|_{B}: f \in V\right\} \geqq \sup \{e(j(f)) / e(f): f \in K\} \\
& =\sup \{T(j)(f): f \in B\}=\|T(j)\|
\end{aligned}
$$

and therefore the mapping $j \mapsto T(j)$ is norm non-increasing. However, this result does not give any information about continuity properties as far as the relativised topologies of $\mathscr{P}$ and $\mathscr{Q}$ are concerned, since it is not linear. However, for $j_{1}, j_{2} \in \mathscr{P}, f_{1}, f_{2} \in K$,

$$
\begin{aligned}
& \left|T\left(j_{1}\right)\left(f_{1}-f_{2}\right)-T\left(j_{2}\right)\left(f_{1}-f_{2}\right)\right| \\
& \quad \leqq\left|e\left(j_{1}\left(f_{1}\right)\right)-e\left(j_{2}\left(f_{1}\right)\right)\right|+\left|e\left(j_{1}\left(f_{2}\right)\right)-e\left(j_{2}\left(f_{2}\right)\right)\right| \\
& \quad \leqq\left\|j_{1}\left(f_{1}\right)-j_{2}\left(f_{1}\right)\right\|_{B}+\left\|j_{1}\left(f_{2}\right)-j_{2}\left(f_{1}\right)\right\|_{B} \\
& \quad \leqq\left\|j_{1}-j_{2}\right\|\left(e\left(f_{1}\right)+e\left(f_{2}\right)\right) .
\end{aligned}
$$

It follows that, for $f \in V$,

$$
\left|T\left(j_{1}\right)(f)-T\left(j_{2}\right)(f)\right| \leqq\left\|j_{1}-j_{2}\right\|\|f\|_{B}
$$

and hence that $j \mapsto T(j)$ is continuous for the relativised uniform topology of $\mathscr{P}$ and the relativised norm topology of $\mathscr{Q}$. Hence, every isotony class is a uniformly closed convex subset of $\mathscr{P}$. Further, since $j \mapsto T(j)$ is affine and maps onto $\mathscr{Q}, A \in E(\mathscr{Q})$ if and only if $T^{-1}(A)$ is a face of $\mathscr{P}$.

For $j_{1}, j_{2} \in \mathscr{P}, j_{1} j_{2}$ is said to be the composition of $j_{1}$ and $j_{2}$ and corresponds to a physical process in which $j_{1}$ follows $j_{2}$. For $f \in K$,

$$
T\left(j_{1} j_{2}\right)(f)=e\left(j_{1} j_{2}(f)\right) \leqq e\left(j_{2}(f)\right)=T\left(j_{2}\right)(f)
$$

and hence, $T\left(j_{1} j_{2}\right) \leqq T\left(j_{2}\right)$.

For the ordering of $\mathfrak{Q}(V)$ defined by $\mathscr{K}, j_{1}, j_{2} \in \mathscr{P}, j_{1} \leqq j_{2}$ if and only if $A\left(\left(j_{2}-j_{1}\right)(f)\right) \geqq 0, \forall f \in K, A \in \mathscr{Q}$ and hence, if and only if $T\left(j j_{2}\right) \geqq T\left(j j_{1}\right)$, $\forall j \in \mathscr{P}$.

Finally, notice that, for $j \in \mathscr{P}, T(j)=j^{*}(e)$. The properties of $\mathscr{Q}, \mathscr{P}$ and $j \mapsto T(j)$ are listed below.

Proposition 3.2. $j \mapsto T(j)$ is an affine mapping from the uniformly closed convex set $\mathscr{P}$ in $\mathfrak{Q}(V)$ onto the $\sigma\left(V^{*}, V\right)$ compact set $\mathscr{Q}$ in $V^{*}$ continuous 
for the relativised uniform topology of $\mathscr{P}$ and the relativised norm topology of 2 and satisfying,

(i) $T(j)=j^{*}(e), \forall j \in \mathscr{P}$.

(ii) For $j_{1}, j_{2} \in \mathscr{P}, T\left(j_{1} j_{2}\right) \leqq T\left(j_{2}\right)$ and $j_{1} \leqq j_{2}$ if and only if $T\left(j j_{1}\right)$ $\leqq T\left(j j_{2}\right), \forall j \in \mathscr{P}$.

(iii) For $A \in \mathscr{Q}, T^{-1}(A)$ is a uniformly closed convex subset of $\mathscr{P}$ and is a face of $\mathscr{P}$ if and only if $A \in E(2)$.

The properties of operations which are likely to be of physical interest fall into two categories, namely those which are shared by all members of an isotony class and those which are not. Physically, the difference between the two categories of properties is that the existence of the former type of property can be determined by experiments which merely count the systems in any state, whilst the latter type of property requires more sophisticated experiments to determine its existence. An example of the former is "under an operation all copies of the system in every state are transmitted", and an example of the latter is "under an operation all copies of the system in every state are transmitted and the beam of systems is rotated through some angle". By merely counting transmitted systems the two patently different properties are indistinguishable.

For $A \in \mathscr{Q}$, define

$$
K_{A}=\{A\}_{0} \cap K, \quad H_{A}=\{e-A\}_{0} \cap K .
$$

For $j \in T^{-1}(A), K_{A}=\{f: f \in K, e(j(f))=0\}, H_{A}=\{f: f \in K, e(j(f))=e(f)\}$. $K_{A}, H_{A}$ are said to be the sets of non-transmitted and transmitted states, respectively, under the operation $j$. Since $\{A\},\{e-A\}$ are positively generated sets in $V^{*}, K_{A}, H_{A}$ are norm closed extremal sets in $K$. Associated with each simple observable $A$, there is a unique complementary simple observable $e-A$ and for $j \in T^{-1}(A), \mathscr{T}_{j}=T^{-1}(e-A)$ is said to be the isotony class of operations complementary to $j$. Since $A \in E(\mathscr{Q})$ if and only if $e-A \in E(\mathscr{Q})$, it follows that $\mathscr{T}_{j}$ is a face of $\mathscr{P}$ if and only $T(j) \in E(\mathscr{Q})$.

Clearly, for $A \in \mathscr{Q}, K_{e-A}=H_{A}, H_{e-A}=K_{A}$ and for $A_{1}, A_{2} \in \mathscr{Q}, A_{1} \leqq A_{2}$ implies that $K_{A_{2}} \subset K_{A_{1}}, H_{A_{1}} \subset H_{A_{2}}$. Some discussion of such properties was given by Davies and Lewis [4] in their remarks on material implication. They defined a notion of material implication for simple observables as follows: $A_{1} \Rightarrow A_{2}$ if and only if $H_{A_{1}} \subset H_{A_{2}}$. As they pointed out, this definition is most reasonable from a physical point of view, although it is weaker in general than that of Mackey [17] whose axioms for quantum mechanics would lead to the definition: $A_{1} \Rightarrow A_{2}$ if and only if $A_{1} \leqq A_{2}$, in this case.

For $j, j^{\prime} \in \mathscr{P}$, since $T\left(j j^{\prime}\right) \leqq T\left(j^{\prime}\right)$ it follows that

$$
K_{T\left(j^{\prime}\right)} \subset K_{T\left(j j^{\prime}\right)}, H_{T\left(j j^{\prime}\right)} \subset H_{T\left(j^{\prime}\right)} .
$$


If $j^{\prime} \in \mathscr{T}_{j^{\prime}}$

and hence,

$$
\begin{aligned}
T\left(j j^{\prime}\right)(f) & =T(j)\left(j^{\prime}(f)\right)=\left(e-T\left(j^{\prime}\right)\right)\left(j^{\prime}(f)\right) \\
& =\left(T\left(j^{\prime}\right)-T\left(j^{\prime 2}\right)\right)(f), \quad \forall f \in K
\end{aligned}
$$

$$
T\left(j j^{\prime}\right)=T\left(j^{\prime}\right)-T\left(j^{\prime 2}\right), T\left(j^{\prime} j\right)=T(j)-T\left(j^{2}\right) .
$$

The following result lists the properties of complementation.

Proposition 3.3. (i) For $A \in \mathscr{Q}, K_{A}, H_{A}$ are norm closed extremal sets in $K$.

(ii) For $A_{1}, A_{2} \in \mathscr{Q}, t \in[0,1]$,

$$
K_{t A_{1}+(1-t) A_{2}}=K_{A_{1}} \cap K_{A_{2}}, H_{t A_{1}+(1-t) A_{2}}=H_{A_{1}} \cap H_{A_{2}} .
$$

(iii) For $A \in \mathscr{2}, K_{e-A}=H_{A}, H_{e-A}=K_{A}$ and for $t \in(0,1)$,

$$
K_{t A+(1-t)(e-A)}=H_{t A+(1-t)(e-A)}=\{0\} .
$$

(iv) For $j \in \mathscr{P}, \mathscr{T}_{j}$ is a uniformly closed convex subset of $\mathscr{P}$ and is a face of $\mathscr{P}$ if and only if $T(j) \in E(\mathscr{Q})$.

(v) For $j \in \mathscr{P}, j^{\prime} \in \mathscr{T}_{j}$,

$$
T\left(j j^{\prime}\right)=T\left(j^{\prime}\right)-T\left(j^{\prime 2}\right), T\left(j^{\prime} j\right)=T(j)-T\left(j^{2}\right) .
$$

An operation $j$ is said to be a transmission if and only if $H_{T(j)}=K$. Physically, $j$ is a transmission if and only if the number of copies of the system in every state is unchanged when operated upon by $j$. Let $\mathscr{P}_{T}$ denote the set of transmissions in $\mathscr{P}$. Clearly $j \in \mathscr{P}_{T}$ if and only if $T(j)=e$ and if and only if $j^{*}(e)=e$. Since $e \in E(2)$ it follows that $\mathscr{P}_{T}$ is a uniformly closed face of $\mathscr{P}$. The properties of transmissions are listed below.

Proposition 3.4. (i) The set $\mathscr{P}_{T}$ of transmissions is a uniformly closed face of $\mathscr{P}$.

(ii) The following conditions on $j \in \mathscr{P}$ are equivalent: $j \in \mathscr{P}_{T} ; K_{T(j)}$ $=\{0\} ; j$ maps $V$ one-one into $V ; j^{*}(e)=e ; j^{\prime} j \in \mathscr{P}_{T}$ some $j^{\prime} \in \mathscr{P}$.

(iii) For $j, j^{\prime} \in \mathscr{P}$,

(a) $j, j^{\prime} \in \mathscr{P}_{T}$ implies $j j^{\prime} \in \mathscr{P}_{T}$, (b) $j \in \mathscr{P}_{T}$ implies $T\left(j j^{\prime}\right)=T\left(j^{\prime}\right)$, (c) $j \in \mathscr{P}_{T}$, $j \leqq j^{\prime}$ implies $j^{\prime} \in \mathscr{P}_{T}$.

Two operations $j, j^{\prime} \in \mathscr{P}$ are said to be weakly commuting when $T\left(j j^{\prime}\right)=T\left(j^{\prime} j\right)$ or alternatively when $j^{*} j^{\prime *}(e)=j^{\prime *} j^{*}(e)$. For $j \in \mathscr{P}$, let

$$
\begin{gathered}
\mathscr{L}_{j}=\left\{j^{\prime}: j^{\prime} \in \mathscr{P}, T\left(j^{\prime} j\right)=0\right\}, \quad \mathscr{R}_{j}=\left\{j^{\prime}: j^{\prime} \in \mathscr{P}, T\left(j j^{\prime}\right)=0\right\} \\
\mathscr{U}_{j}=\mathscr{L}_{j} \cap \mathscr{R}_{j} .
\end{gathered}
$$

$\mathscr{L}_{j}, \mathscr{R}_{j}, \mathscr{U}_{j}$ are said to be the sets of operations, nullifying $j$, nullified by $j$, and disjoint from $j$ respectively. $\mathscr{L}_{j}, \mathscr{R}_{j}, \mathscr{U}_{j}$ are uniformly closed faces of 
$\mathscr{P}$ and, for $j^{\prime} \in \mathscr{L}_{j}, j_{1} \in T^{-1}\left(T\left(j^{\prime}\right)\right)$,

$$
T\left(j_{1} j\right)(f)=T\left(j_{1}\right)(j(f))=T\left(j^{\prime}\right)(j(f))=T\left(j^{\prime} j\right)(f)=0
$$

$\forall f \in K$ and hence $j_{1} \in \mathscr{L}_{j}$. It follows that $T^{-1}\left(T\left(j^{\prime}\right)\right) \subset \mathscr{L}_{j}$. Prop. 3.3 (v) shows that $j^{\prime} \in \mathscr{T}_{j} \cap \mathscr{L}_{j}$ if and only if $T(j)=T\left(j^{2}\right)$ and $j^{\prime} \in \mathscr{T}_{j} \cap \mathscr{R}_{j}$ if and only if $T\left(j^{\prime}\right)=T\left(j^{\prime 2}\right)$. From this remark it follows that $\mathscr{T}_{j} \cap \mathscr{L}_{j} \neq \emptyset$ if and only if $T(j)=T\left(j^{2}\right)$ and in this case $\mathscr{T}_{j} \subset \mathscr{L}_{j}$ and $\mathscr{T}_{j} \cap \mathscr{R}_{j} \neq \emptyset$ if and only if there exists $j^{\prime} \in \mathscr{T}_{j}$ such that $T\left(j^{\prime}\right)=T\left(j^{\prime 2}\right)$ and in this case, $T^{-1}(T(j)) \subset \mathscr{L}_{j^{\prime}}$.

If $\mathscr{T}_{j} \cap \mathscr{L}_{j} \neq \emptyset, T(j)=T\left(j^{2}\right)$ and, $\forall f \in K, j(f) \in H_{T(j)}$ and hence if $j \neq \mathbf{0}$,

$$
\|j\| \geqq\|T(j)\|=\sup \{T(j)(f) / e(f): f \in K\} \geqq 1 .
$$

It follows that $\|T(j)\|=\|j\|=1$. Hence, the following important result has been proved.

Proposition 3.5. (i) $\mathscr{L}_{j}, \mathscr{R}_{j}, \mathscr{U}_{j}$ are uniformly closed faces of $\mathscr{P}$.

(ii) $j^{\prime} \in \mathscr{L}_{j}$ implies $T^{-1}\left(T\left(j^{\prime}\right)\right) \subset \mathscr{L}_{j}$.

(iii) $\mathscr{P} \mathscr{L}_{j} \subset \mathscr{L}_{j}, \mathscr{R}_{j} \mathscr{P} \subset \mathscr{R}_{j}, \mathscr{P} \mathscr{U}_{j} \subset \mathscr{U}_{j}, \mathscr{U}_{j} \mathscr{P} \subset \mathscr{U}_{j}$ and if $j_{1} \leqq j_{2} \in \mathscr{L}_{j}, \mathscr{R}_{j}$ or $\mathscr{U}_{j}, j_{1} \in \mathscr{L}_{j}, \mathscr{R}_{j}$ or $\mathscr{U}_{j}$ respectively.

(iv) For $j \in \mathscr{P}, \mathscr{T}_{j} \cap \mathscr{L}_{j} \neq \emptyset$ if and only if $T(j)=T\left(j^{2}\right)$ and in this case $\mathscr{T}_{j} \subset \mathscr{L}_{j}$ and if $j \neq \mathbf{0}, H_{T(j)} \neq\{0\},\|j\|=\|T(j)\|=1, \mathscr{T}_{j} \cap \mathscr{R}_{j} \neq \emptyset$ if and only if there exists $j^{\prime} \in \mathscr{T}_{j}$ such that $T\left(j^{\prime}\right)=T\left(j^{\prime 2}\right)$ and in this case $T^{-1}(T(j)) \subset \mathscr{L}_{j}$, and if $j \neq \mathbf{1}, K_{T(j)} \neq\{0\},\left\|j^{\prime}\right\|=\left\|T\left(j^{\prime}\right)\right\|=1 . \mathscr{T}_{j} \cap \mathscr{U}_{j} \neq \emptyset$ if and only if $T(j)=T\left(j^{2}\right)$ and there exists $j^{\prime} \in \mathscr{T}_{j}$ such that $T\left(j^{\prime}\right)=T\left(j^{\prime 2}\right)$ and in this case $\mathscr{T}_{j} \subset \mathscr{L}_{j}, \mathscr{T}_{j^{\prime}} \subset \mathscr{L}_{j^{\prime}}$ and if $j \neq \mathbf{0}$ or $\mathbf{1}, H_{T(j)} \neq\{0\}, K_{T(j)} \neq\{0\}\|j\|=\left\|j^{\prime}\right\|$ $=\|T(j)\|=\left\|T\left(j^{\prime}\right)\right\|=1$.

The set of operations $j$ such that $\mathscr{T}_{j} \cap \mathscr{U}_{j} \neq \emptyset$ form an important class. Following Davies and Lewis [4], such operations are said to be repeatable and the set of all such operations is denoted by $\mathscr{P}_{R}$. It follows from Propn. 3.5 (iv) that $j \in \mathscr{P}_{R}$ if and only if there exists $j^{\prime} \in \mathscr{P}$ such that

(i) $T(j)+T\left(j^{\prime}\right)=e$,

(ii) $T(j)=T\left(j^{2}\right), T\left(j^{\prime}\right)=T\left(j^{\prime 2}\right)$,

and (ii) can alternatively be replaced by

(ii) $T\left(j j^{\prime}\right)=T\left(j^{\prime} j\right)=0$.

The symmetric nature of the definition shows that $j \in \mathscr{P}_{R}$ if and only if $j^{\prime} \in \mathscr{P}_{R}$. In future, $j^{\prime}$ will be said to be a repeatable operation complementary to $j \in \mathscr{P}_{R}$. Physically, repeatable operations are those for which a second application yields the same transmission probabilities on all states as did the first and which possesses a complementary operation with the same property. Two points now arise. First, as was pointed out in [4], it may be thought that repeatable operations are those which produce states according to Von Neumann's measurement theory [21]. Secondly, since, for $j \in \mathscr{P}_{R}, j \neq \mathbf{0},\|T(j)\|=1$ and it might be thought that 
repeatable operations give rise to extreme simple observables. The next result shows that this is the case if a further condition is imposed.

Proposition 3.6. Let $j \in \mathscr{P}_{R}$ and let $j^{\prime}$ be a complementary repeatable operation. If one of the following equivalent conditions holds, then $T(j) \in E(2):$

(a) For $j_{1} \in \mathscr{P}, T\left(j_{1} j\right)=T(j), T\left(j_{1} j^{\prime}\right)=0$ implies $T\left(j_{1}\right)=T(j)$.

(b) For $j_{2} \in \mathscr{P}, T\left(j_{2} j\right)=0, T\left(j_{2} j^{\prime}\right)=T\left(j^{\prime}\right)$ implies $T\left(j_{2}\right)=T\left(j^{\prime}\right)$.

Proof. First, conditions (a) and (b) are shown to be equivalent. Suppose (a) holds and let $j_{2} \in \mathscr{P}$ satisfy $T\left(j_{2} j\right)=0, T\left(j_{2} j^{\prime}\right)=T\left(j^{\prime}\right)$ Then, if $T\left(j_{1}\right)=e-T\left(j_{2}\right)$,

$$
T\left(j_{1} j\right)(f)=\left(e-T\left(j_{2}\right)\right)(j(f))=\left(T(j)-T\left(j_{2} j\right)\right)(f)=T(j)(f),
$$

$\forall f \in K$, and hence, $T\left(j_{1} j\right)=T(j)$. Similarly $T\left(j_{1} j^{\prime}\right)=0$ and therefore, $T\left(j_{1}\right)=T(j), T\left(j_{2}\right)=e-T(j)=T\left(j^{\prime}\right)$ and (b) holds. A similar argument shows that (b) implies (a).

Let $A_{1}, A_{2} \in \mathscr{Q}, t \in(0,1)$ and suppose that for $j \in \mathscr{P}_{R}$,

Then,

$$
T(j)=t A_{1}+(1-t) A_{2} .
$$

$$
e(j(f))=T(j)(f)=t A_{1}(f)+(1-t) A_{2}(f), \forall f \in K,
$$

and therefore, replacing $f$ by $j^{\prime}(f), j(f)$ respectively,

$$
\begin{gathered}
0=T\left(j j^{\prime}\right)(f)=e\left(j j^{\prime}(f)\right)=t A_{1}\left(j^{\prime}(f)\right)+(1-t) A_{2}\left(j^{\prime}(f)\right), \\
T(j)(f)=T\left(j^{2}\right)(f)=e\left(j^{2}(f)\right)=t A_{1}(j(f))+(1-t) A_{2}(j(f)),
\end{gathered}
$$

$\forall f \in K$. It follows from (3.9) that $A_{i}\left(j^{\prime}(f)\right)=0, i=1,2, \forall f \in K$, and, since $A_{i}(j(f)) \leqq e(j(f))=T(j)(f), i=1,2, \forall f \in K$, from (3.10) that $A_{i}(j(f))$ $=T(j)(f), i=1,2, \forall f \in K$. If (a) holds, choosing $j_{1}, j_{2} \in \mathscr{P}$ such that $T\left(j_{i}\right)=A_{i}, i=1,2$, it follows that $A_{i}=T(j), i=1,2$. Therefore, $T(j) \in E(\mathscr{Q})$. This completes the proof.

Suppose that $j \in \mathscr{P}_{R}$ has complementary repeatable operation $j^{\prime}$ and let $j_{1} \in \mathscr{P}$ satisfy $T\left(j_{1} j\right)=T(j), T\left(j_{1} j^{\prime}\right)=0$. Then, physically, a measurement of the simple observable defined by $j_{1}$ on the states obtained by operating with $j, j^{\prime}$ produces an effect indistinguishable from that obtained by a second measurement of $j$ and $j^{\prime}$. In a sense, therefore conditions (a) or (b) of Prop. 3.6 can be interpreted as a physical condition preventing the existence of superselection rules for measurements of $T(j)$ and $T\left(j^{\prime}\right)$.

Notice that conditions (a), (b) of Prop. 3.6 appear to differ from condition SR 3 of [4]. For $j \in \mathscr{P}_{R}$ with complementary repeatable operation $j^{\prime}$, this condition may be stated,

(c) For $A \in \mathscr{Q}, A(j(f))=A\left(j^{\prime}(f)\right)=0, \forall f \in K$, implies $A=0$. The following result shows that conditions (a) and (b) imply that a slightly weakened version of $(\mathrm{c})$ is true. 
Proposition 3.7. Let $j \in \mathscr{P}_{R}$ have complementary repeatable operation $j^{\prime}$ and let $j, j^{\prime}$ satisfy condition (a) or (b) of Prop. 3.6. Then, $j, j^{\prime}$ satisfy the following condition.

(d) For $A \in \mathscr{Q}, A \leqq T(j), A(j(f))=A\left(j^{\prime}(f)\right)=0, \forall f \in K$, implies $A=0$.

Proof. Suppose, $j, j^{\prime}$ satisfy condition (a) of Prop. 3.6 and let $A \in \mathscr{Q}$ be such that $A \leqq T(j), A(j(f))=A\left(j^{\prime}(f)\right)=0, \forall f \in K$. Let $j_{1} \in \mathscr{P}$ satisfy $T\left(j_{1}\right)=T(j)-A$. Then,

$$
\begin{aligned}
T\left(j_{1} j\right)(f) & =(T(j)-A)(j(f))=T\left(j^{2}\right)(f)=T(j)(f), \forall f \in K, \\
T\left(j_{1} j^{\prime}\right)(f) & =(T(j)-A)\left(j^{\prime}(f)\right)=T\left(j j^{\prime}\right)(f)=0, \quad \forall f \in K
\end{aligned}
$$

and therefore $T\left(j_{1}\right)=T(j), A=0$. Therefore (d) above holds and since (a) and (b) are equivalent (b) also implies (d).

In the following, a repeatable operation $j$ with complementary repeatable operation $j^{\prime}$ satisfying conditions (a) and (b) or (c) is said to be (a) or (c)-repeatable respectively.

The properties of operations which have so far been discussed have been such that their existence may be determined by a consideration of transmission probabilities alone. Next, an examination is made of a wider class of properties of operations. For example, $j, j^{\prime}$ are said to commute strongly if $j j^{\prime}=j^{\prime} j$.

Recall that the set $\mathscr{P}_{T}$ of transmissions coincides with the set of operations mapping $K$ one-one into itself. A transmission $j$ such that $j(K)=K$ is said to be a symmetry of the system. Clearly, the set $\mathscr{P}_{S}$ of symmetries forms a group with identity 1 .

At this stage it is convenient to introduce a further set of states associated with any operation. For $j \in \mathscr{P}$, let

$$
G_{j}=\{f: f \in K, j(f)=f\} .
$$

Then, $G_{j}$ is said to be the set of unchanged states under the operation $j$. $G_{j}$ is clearly a uniformly closed convex subset of $H_{T(j)}$ and $G_{j}=K$ if and only if $j=\mathbf{1}$. Before proceeding to a discussion of the class of operations for which $G_{j}$ has the greatest importance, the properties of symmetries are listed.

Proposition 3.8. (i) $\mathscr{P}_{S}$ is a group with identity 1.

(ii) $F$ or $j \in \mathscr{P}_{S}, j^{\prime} \in \mathscr{P}$,

$$
K_{T\left(j^{-1} j^{\prime} j\right)}=j^{-1}\left(K_{T\left(j^{\prime}\right)}\right), G_{j^{-1} j^{\prime} j}=j^{-1}\left(G_{j^{\prime}}\right)
$$

and in particular, for $j^{\prime} \in \mathscr{P}_{T}, j^{-1} j^{\prime} j \in \mathscr{P}_{T}$.

At the other extreme from the group of symmetries of the system is the set of operations which act only as filters. An operation $j$ is said to be a filtering if and only if $G_{j}=H_{T(j)}$. Physically, any state which is 
unaltered numerically under $j$ is unchanged. Let $\mathscr{P}_{F}$ denote the set of filterings in $\mathscr{P}$. Clearly $\mathscr{P}_{F}$ is convex and if $j_{1}, j_{2} \in \mathscr{P}_{F}, j_{1} j_{2} \in \mathscr{P}_{F}$.

Let $j \in \mathscr{P}_{F}, j^{\prime} \in \mathscr{T}_{j}$. Then, Prop. 3.3 (v) shows that $T\left(j^{\prime} j\right)=T(j)-T\left(j^{2}\right)$ and $f \in K_{T\left(j^{\prime} j\right)}$ if and only if $j(f) \in H_{T(j)}=G_{j}$. It follows that $f \in K_{T\left(j^{\prime} j\right)}$ if and only if $j^{2}(f)=j(f)$. Similarly, for $j \in \mathscr{P}, j^{\prime} \in \mathscr{T}_{j} \cap \mathscr{P}_{F}, f \in K_{T\left(j j^{\prime}\right)}$ if and only if $j^{\prime 2}(f)=j^{\prime}(f)$. Using Prop. 3.5 (iv) it follows that for $j \in \mathscr{P}_{F}$, $\mathscr{T}_{j} \cap \mathscr{L}_{j} \neq \emptyset$ if and only if $j=j^{2}$ and for $j \in \mathscr{P}, \mathscr{T}_{j} \cap \mathscr{R}_{j} \cap \mathscr{P}_{F} \neq \emptyset$ if and only if there exists $j^{\prime} \in \mathscr{T}_{j} \cap \mathscr{P}_{F}$ such that $j^{\prime}=j^{\prime 2}$.

The properties of $\mathscr{P}_{F}$ are summarised in

Proposition 3.9. (i) $\mathscr{P}_{F}$ is a convex subset of $\mathscr{P}$ such that, for $j, j^{\prime} \in \mathscr{P}_{F}$, $j j^{\prime} \in \mathscr{P}_{F}$.

(ii) $j \in \mathscr{P}_{F}, j^{\prime} \in \mathscr{T}_{j}$ implies that $K_{T\left(j^{\prime} j\right)}=\left\{f: f \in K, j^{2}(f)=j(f)\right\}, j \in \mathscr{P}$, $j^{\prime} \in \mathscr{T}_{j} \cap \mathscr{P}_{F}$ implies that $K_{T\left(j j^{\prime}\right)}=\left\{f: f \in K, j^{\prime 2}(f)=j^{\prime}(f)\right\}$.

(iii) $\mathscr{P}_{F} \cap \mathscr{P}_{T}=\{\boldsymbol{1}\}$, and $j^{-1} \mathscr{P}_{F} j \subset \mathscr{P}_{F}, \forall j \in \mathscr{P}_{S}$.

(iv) For $j \in \mathscr{P}_{F}, \mathscr{T}_{j} \cap \mathscr{L}_{j} \neq \emptyset$ if and only if $j=j^{2}$, for $j \in \mathscr{P}, \mathscr{T}_{j} \cap \mathscr{R}_{j} \cap \mathscr{P}_{F}$ $\neq \emptyset$ if and only if there exists $j^{\prime} \in \mathscr{T}_{j} \cap \mathscr{P}_{F}$ such that $j^{\prime}=j^{\prime 2}$ and for $j \in \mathscr{P}_{F}$, $\mathscr{T}_{j} \cap \mathscr{U}_{j} \cap \mathscr{P}_{F} \neq \emptyset$ if and only if $j=j^{2}$ and there exists $j^{\prime} \in \mathscr{T}_{j} \cap \mathscr{P}_{F}$ such that $j^{\prime}=j^{\prime 2}$.

Following [4], an (a) or (c)-repeatable operation $j$ with complementary repeatable operation $j^{\prime}$ is said to be strongly (a) or (c)-repeatable if and only if $j, j^{\prime} \in \mathscr{P}_{F}$. The sets of all such operations will be denoted by $\mathscr{P}_{S R a}$ and $\mathscr{P}_{S R c}$ respectively. Summarising, $j \in \mathscr{P}_{S R a}$ or $\mathscr{P}_{S R c}$ if and only if there exists $j^{\prime} \in \mathscr{P}$ such that

(i) $T(j)+T\left(j^{\prime}\right)=e$,

(ii) $T(j)=T\left(j^{2}\right), T\left(j^{\prime}\right)=T\left(j^{\prime 2}\right)$,

(iii) $T(j)(f)=e(f)$ implies $j(f)=f, T\left(j^{\prime}\right)(f)=e(f)$, implies $j^{\prime}(f)=f$,

(iv) (a) if $j_{1} \in \mathscr{P}$ satisfies $T\left(j_{1} j\right)=T(j), T\left(j_{1} j^{\prime}\right)=0$, then $T\left(j_{1}\right)=T(j)$, or equivalently,

(b) if $j_{1} \in \mathscr{P}$ satisfies $T\left(j_{1} j\right)=0, T\left(j_{1} j^{\prime}\right)=T\left(j^{\prime}\right)$, then $T\left(j_{1}\right)=T\left(j^{\prime}\right)$, or,

(c) if $A \in \mathscr{Q}$ satisfies $A(j(f))=A\left(j^{\prime}(f)\right)=0, \forall f \in K$, then $A=0$, respectively.

Immediate consequences of the definition are that $j j^{\prime}=j^{\prime} j=0, j=j^{2}$ and $j^{\prime}=j^{\prime 2}$.

For $j \in \mathscr{P}, \mathbf{1}-j$ is a bounded though not necessarily positive element of $\mathfrak{L}(V)$. When, relative to the ordering of $\mathfrak{L}(V)$ by $\mathscr{K}, j \leqq 1, j$ is said to be a reflection. In this case, it is clear that $\mathbf{1}-j \in \mathscr{P}$ and that $T(\mathbf{1}-j)$ $=e-T(j)$, which implies that $1-j \in \mathscr{T}_{j}$. Let $\mathscr{P}_{W}$ denote the set of reflections in $\mathscr{P}$. Physically, an operation $j$ produces a new state $j(f)$ from a state $f$ and any complementary operation $j^{\prime}$ produces a new state $j^{\prime}(f)$ from $f$, in such a way that the total number of systems in $f$ is preserved. Reflections $j$ are distinguished among all operations in that there exists a unique complementary operation $1-j$ such that the mixture of the two 
states obtained by operating with $j$ and $\mathbf{1}-j$ on $f$ is $f$ itself. $j$ may be thought of as producing a transmitted state $j(f)$ and a reflected state $f-j(f)$.

Clearly $\mathscr{P}_{W}$ is a uniformly closed convex subset of $\mathscr{P}$ and the mapping $j \mapsto \mathbf{1}-j$ is a uniformly continuous affine automorphism of $\mathscr{P}_{W}$. For $j \in \mathscr{P}_{W}$, suppose $f \in H_{T(j)}$. Then, $e((\mathbf{1}-j) f)=0$ which implies that $j(f)$ $=f, f \in G_{j}$. It follows that $j \in \mathscr{P}_{F}$. For $j \in \mathscr{P}_{W}$, Propn. 3.9 (iv) shows that $\mathscr{T}_{j} \cap \mathscr{L}_{j} \neq \emptyset$ if and only if $j=j^{2}$ and hence, if and only if $j(\mathbf{1}-j)=0$ or $(\mathbf{1}-j) j=0$. Let $j_{1} \in \mathscr{P}$ satisfy $T\left(j_{1} j\right)=T(j), T\left(j_{1}(\mathbf{1}-j)\right)=0$. Then clearly $T\left(j_{1}\right)=T(j)$ and similarly if $A \in \mathscr{Q}$ satisfies $A(j(f))=A((1-j) f)=0$, $\forall f \in K$, then $A=0$. The properties of $\mathscr{P}_{W}$ are listed below.

Proposition 3.10. (i) $\mathscr{P}_{W}$ is a uniformly closed convex subset of $\mathscr{P}$ and the mapping $j \mapsto \mathbf{1}-j$ is a uniformly continuous affine automorphism of $\mathscr{P}_{W}$.

(ii) $\mathscr{P}_{W} \subset \mathscr{P}_{F}$.

(iii) The following conditions on $j \in \mathscr{P}_{W}$ are equivalent: $\mathscr{T}_{j} \cap \mathscr{L}_{j} \neq \emptyset$; $\mathscr{T}_{j} \cap \mathscr{U}_{j} \neq \emptyset ; j=j^{2}$.

(iv) If $j \in \mathscr{P}_{W}, j=j^{2}$ then $j \in \mathscr{P}_{S R a} \cap \mathscr{P}_{S R c}$.

(v) $j, j^{\prime} \in \mathscr{P}_{W}$ implies $j j^{\prime} \in \mathscr{P}_{W}, j^{-1} \mathscr{P}_{W} j \subset \mathscr{P}_{W}, \forall j \in \mathscr{P}_{S}$.

An operation $j$ in $P_{W}$ satisfying $j=j^{2}$ is said to be a strongly repeatable reflection and the set of all such operations is denoted by $\mathscr{P}_{S R W}$. Such operations are clearly of a very special nature. It should be remarked that elements of $\mathscr{P}_{S R W}$ are " $L$-projections" in the sense of [2] and have also been studied by Gerzon [12].

It is not proposed to make a complete study of the structure of $\mathscr{P}_{\text {SRW }}$ here. However, certain remarks are required for the successive sections.

For $j \in \mathscr{P}_{\text {SRW }}, f \in K$,

$$
f=j(f)+(\mathbf{1}-j) f
$$

is a decomposition into elements $j(f) \in H_{T(j)}=G_{j},(1-j)(f) \in K_{T(j)}$. Suppose $f=f_{1}+f_{2}$ is a further such decomposition. Then,

$$
j(f)=j\left(f_{1}\right)=f_{1},(\mathbf{1}-j)(f)=f_{1}+f_{2}-f_{1}=f_{2}
$$

and hence the decomposition (3.11) is unique. Suppose $H$ is an extremal set in $K$ such that $H \cap H_{T(j)}=\{0\}$. Then, for $f \in H$

$$
f=j(f)+(\mathbf{1}-j) f, \quad j(f) \in H_{T(j)},(\mathbf{1}-j)(f) \in K_{T(j)} .
$$

Since $H$ is extremal, $j(f),(\mathbf{1}-j)(f) \in H$ and hence, $j(f) \in H \cap H_{T(j)}$ which implies that $j(f)=0$ and $H \subset K_{T(j)}$. It follows that the complementary set $H^{\prime}$ of $H$ is contained in $K_{T(j)}$. However, $K_{T(j)}$ is an extremal set in $K$ such that $K_{T(j)} \cap H_{T(j)}=\{0\}$ and therefore $K_{T(j)} \subset H^{\prime}$. Hence $H^{\prime}=K_{T(j)}$ and $H$ is a split extremal set. 
Conversely, suppose $j \in \mathscr{P}_{F}$ and every $f \in K$ has a decomposition

$$
f=f_{1}+f_{2}, \quad f_{1} \in H_{T(j)}=G_{j}, f_{2} \in K_{T(j)} .
$$

Then, $j(f)=f_{1}$ and $f-j(f)=f_{2} \in K$. Therefore $j \in \mathscr{P}_{W}$ and

$$
j^{2}(f)=j\left(f_{1}\right)=f_{1}=j(f)
$$

which implies that $j=j^{2}$ and $j \in \mathscr{P}_{S R W}$. It follows then that $H_{T(j)}$ is a split extremal set with complementary set $K_{T(j)}$ and that the decomposition (3.12) is unique.

Next suppose that $H$ is any split extremal set in $K$ and for $f \in K$, define $j(f)=f_{1}$ where $f=f_{1}+f_{2}$ is the unique decomposition of $f$ into elements of $H, H^{\prime}$ respectively. Then, clearly, $j$ is an affine mapping from $K$ into $H$ and hence extends to a positive linear mapping on $V$. For $f \in K$,

$$
e(j(f))=e\left(f_{1}\right) \leqq e(f)
$$

and therefore $j \in \mathscr{P}$. Further, $f-j(f) \in K, \forall f \in K$ which implies that $j \in \mathscr{P}_{W}$. Clearly, $j=j^{2}$ and hence $j \in \mathscr{P}_{S R W}$. Finally,

$$
H_{T(j)}=G_{j}=\{f: f \in K, j(f)=f\} \subset H
$$

and if $f \in H_{T(j)}, f=f_{1}+f_{2}, f_{1} \in H, f_{1} \in H, f_{2} \in H^{\prime}, j(f)=f$ implies $f_{2}=0$. It follows that $H_{T(j)}=H$ and therefore the mapping $j \mapsto H_{T(j)}$ sends $\mathscr{P}_{S R W}$ onto the set of all split extremal sets in $K$, and the uniqueness of the decomposition (3.11) shows that the mapping is one-one.

Proposition 3.11. (i) There exists a one-one correspondence $j \mapsto H_{T(j)}$ between the set $\mathscr{P}_{S R W}$ and the set of all split extremal sets in $K$.

(ii) If $j \in \mathscr{P}_{F}$ satisfies the condition that every element $f \in K$ has a decomposition $f=f_{1}+f_{2}, f_{1} \in H_{T(j)}, f_{2} \in K_{T(j)}$ then $j \in \mathscr{P}_{S R W}$.

It is obvious from the proof of Prop. 3.11 that the mapping $j \mapsto H_{T(j)}$ has far more properties than have been stated above. A list of some of the properties is given below. The proofs which are not obvious can be found in $[1,2,12]$.

(i) For each split extremal set $H, H^{\prime}$ is a split extremal set and if $H=H_{T(j)}, H^{\prime}=H_{T(\mathbf{1}-j)}$.

(ii) Every split extremal set is norm closed.

(iii) For split extremal sets $H_{1}, H_{2}, H_{1} \cap H_{2}, \operatorname{conv}\left(H_{1} \cup H_{2}\right)$ are split extremal sets and if $H_{1}=H_{T\left(j_{1}\right)}, H_{2}=H_{T\left(j_{2}\right)}, H_{1} \cap H_{2}=H_{T\left(j_{1} j_{2}\right)}=H_{T\left(j_{2} j_{1}\right)}$, $\operatorname{conv}\left(H_{1} \cup H_{2}\right)=H_{T\left(j_{1}+j_{2}-j_{1} j_{2}\right)}$. Hence, for $j_{1}, j_{2} \in \mathscr{P}_{S R W}, j_{1} j_{2}=j_{2} j_{1}, j_{1}+j_{2}$ $-j_{1} j_{2} \in \mathscr{P}_{S R W}$.

It was remarked above, that $\mathscr{P}_{S R W}$ forms a very limited class of operations. The following result gives some idea how limited it is. 
Proposition 3.12. For $j \in \mathscr{P}_{S R W}, H_{T(j)}$ is the set of states of a restriction of the system.

Proof. For convenience, let $H=H_{T(j)}, H^{\prime}=K_{T(j)}, F=H \cap B, F^{\prime}=H^{\prime}$ $\cap B, L=\{f: f \in V, j(f)=f\}, L^{\prime}=\{f: f \in V, j(f)=0\}$. Then clearly $H-H$ $C L$ and conversely, suppose $f \in L, f=f_{1}-f_{2}, f_{1}, f_{2} \in K$. Then, $f=j(f)$ $=j\left(f_{1}\right)-j\left(f_{2}\right) \in H-H$. Hence, $H-H=L$ and similarly $H^{\prime}-H^{\prime}=L^{\prime}$. It follows that $(L, F)$ is a base norm space with cone $H$ and clearly, $L, H$ and $F$ are all norm closed in $V$. The base norm $\|\cdot\|_{F}$ on $L$ is defined by

$$
\|f\|_{F}=\inf \left\{e\left(f_{1}\right)+e\left(f_{2}\right): f=f_{1}-f_{2}, f_{1}, f_{2} \in H\right\}, \quad \forall f \in L
$$

and the base norm in $(V, B)$ is defined by

$$
\|f\|_{B}=\inf \left\{e\left(f_{1}\right)+e\left(f_{2}\right): f=f_{1}-f_{2}, f_{1}, f_{2} \in K\right\}, \quad \forall f \in V .
$$

For $f_{1}, f_{2} \in K$, there exist uniquely $f_{11}, f_{21} \in H, f_{12}, f_{22} \in H^{\prime}$ such that

$$
f_{1}=f_{11}+f_{12}, f_{2}=f_{21}+f_{22} \text {. }
$$

If $f=f_{1}-f_{2} \in L$,

$$
f_{11}-f_{21}=j(f)=f=f_{11}+f_{12}-f_{21}-f_{22}
$$

and hence $f_{12}=f_{22}$. Therefore,

$$
e\left(f_{1}\right)+e\left(f_{2}\right)=e\left(f_{11}\right)+e\left(f_{21}\right)+2 e\left(f_{22}\right)
$$

$f_{11}, f_{21} \in H, f_{22} \in H^{\prime}, f=f_{11}-f_{21}$. It follows that

$$
\begin{aligned}
\|f\|_{B} & =\inf \left\{e\left(g_{1}\right)+e\left(g_{2}\right)+2 e(g): f=g_{1}-g_{2}, g_{1}, g_{2} \in H, g \in H^{\prime}\right\} \\
& =\|f\|_{F} .
\end{aligned}
$$

Therefore, $(L, F)$ is a complete base norm space with norm closed cone $H$ and base norm identical to the norm as a closed subspace of $(V, B)$. Hence condition (i) of Postulate 6 holds. Notice that similar remarks apply to $\left(L^{\prime}, F^{\prime}\right)$.

Let $\tilde{A}$ be an affine functional on $H$ such that $0 \leqq A(f) \leqq e(f), \forall f \in H$ and define $A$ on $K$ by $A(f)=\tilde{A}(j(f))$. Then, clearly $A$ is an affine functional on $K$ such that $0 \leqq A(f) \leqq e(f), \forall f \in K$ and $A(f)=\tilde{A}(f), \forall f \in H$. This proves (ii) of Postulate 6 .

The dual space $\left(L^{\prime *}, e_{F^{\prime}}\right)$ of $\left(L^{\prime}, F^{\prime}\right)$ is a complete order unit space Archimedean ordered by $H^{\prime *}$ and as a Banach space $L^{*}$ may be identified with $V^{*} / L^{0}$ by means of the identification

$$
\phi(T)(f)=T(f), \quad \forall T \in V^{*}, \quad \forall f \in L^{\prime},
$$

where $\phi: V^{*} \rightarrow V^{*} / L^{0}$ is the canonical mapping. If $\phi\left(K^{*}\right)=K^{*} / L^{0}$ is the quotient cone in $V^{*} / L^{\prime 0}$, since $K^{*}$ generates $V^{*}, K^{*} / L^{\prime 0}$ generates $V^{*} / L^{\prime}$. 
For $\phi(T) \in K^{*} / L^{\prime 0}, \phi(T)(f) \geqq 0, \forall f \in H^{\prime}$ and hence $\phi(T) \in H^{\prime *}$. Conversely, for $\tilde{T} \in H^{\prime *}$ define $T$ on $V$ by $T(f)=\tilde{T}((1-j) f), \forall f \in V$. Then, $T \in K^{*}, \phi(T)=\tilde{T}$ and hence $H^{*}=K^{*} / L^{\prime}$.

In order to show that $H^{0}$ is positively generated, let $T \in V^{*}, T(f)$ $=0, \forall f \in L$ and $T^{\prime}=T \mid L^{\prime}$. Then, from the result above, since $K^{*} / L^{\prime 0}$ generates $V^{*} / L^{\prime 0}$, there exists $T_{1} \in K^{*}$ such that $\phi\left(T_{1}\right) \geqq T^{\prime}$ or equivalently $\quad T_{1}(f) \geqq T^{\prime}(f)=T(f), \quad \forall f \in H^{\prime}$. Define $T_{2} \in V^{*}$ by $T_{2}(f)$ $=T_{1}((1-j) f), \forall f \in V$. Then $T_{2} \in K^{*} \cap H^{0}$, and for $f \in K$,

$$
\begin{aligned}
T_{2}(f)-T(f) & =T_{1}((\mathbf{1}-j) f)-T(f) \\
& =T_{1}((\mathbf{1}-j) f)-T((\mathbf{1}-j) f)
\end{aligned}
$$

since $T(f)=0, \forall f \in H$,

$$
=T_{1}((\mathbf{1}-j) f)-T^{\prime}((\mathbf{1}-j) f) \geqq 0 .
$$

Hence $T_{2}-T \in K^{*} \cap H^{0}, T=T_{2}-\left(T_{2}-T\right) \in K^{*} \cap H^{0}-K^{*} \cap H^{0}$ which implies that $H^{0}$ is positively generated. This completes the proof.

Hence, the effect of any element of $\mathscr{P}_{S R W}$ is to produce the set of states of a restriction of the system. A class of operations likely to be of some physical significance is the set of elements $j \in \mathscr{P}$ such that for each split extremal set $H$ of $K, j(H) \subset H$. Such operations will be said to be strong operations. The following result shows how they may be characterized.

Proposition 3.13. For $j \in \mathscr{P}, j j^{\prime}=j^{\prime} j, \forall j^{\prime} \in \mathscr{P}_{S R W}$ if and only if for each split extremal set $H$ in $K, j(H) \subset H$.

Proof. Suppose $j \in \mathscr{P}$ satisfies $j j^{\prime}=j^{\prime} j, \forall j^{\prime} \in \mathscr{P}_{S R W}$. Let $H$ be a split extremal set in $K$ and let $j^{\prime} \in \mathscr{P}_{S R W}$ be such that $H=H_{T\left(j^{\prime}\right)}$. For $f \in H$, let $j(f)=f_{1}+f_{2}, f_{1} \in H, f_{2} \in H^{\prime}$. Then,

$$
f_{1}=j^{\prime} j(f)=j j^{\prime}(f)=j(f)=f_{1}+f_{2}
$$

which implies that $f_{2}=0, j(f) \in H$.

Conversely, suppose $j \in \mathscr{P}$, satisfies $j(H) \subset H$ for each split extremal set $H$ in $K$. Let $j^{\prime} \in \mathscr{P}_{S R W}$ and let $H=H_{T\left(j^{\prime}\right)}$. For $f \in K$, let $f=f_{1}+f_{2}$, $f_{1} \in H, f_{2} \in H^{\prime}$. Then, $j\left(f_{1}\right) \in H, j\left(f_{2}\right) \in H^{\prime}$ and

$$
j^{\prime} j(f)=j\left(f_{1}\right)=j j^{\prime}(f) .
$$

Therefore $j j^{\prime}=j^{\prime} j$ and the proof is complete.

As was remarked above, the set of split extremal sets in $K$ define a subset of the set of restrictions of the corresponding physical system and for each restriction corresponding to a split extremal set $H$ a complementary restriction is defined by the complementary split extremal 
set $H^{\prime}$. As was pointed out in [7], this is a desirable property for restrictions to possess. In the following sections, restrictions corresponding to split extremal sets are called strong restrictions.

\section{$\S 4$. The Von Neumann Algebra Model}

For details of the theory of Von Neumann algebras the reader is referred to $[5,6,9,18]$. Let $\mathfrak{B}$ be a Von Neumann algebra acting on the Hilbert space $X$ and let $e$, the identity in $\mathfrak{B}$, be also the identity in the algebra $\mathfrak{L}(X)$ of bounded linear operators on $X$. Let $\mathfrak{B}_{*}$ be the pre-dual of $\mathfrak{B}, V(\mathfrak{B})$ the space of hermitean elements of $\mathfrak{B}_{*}, K(\mathfrak{B})$ the cone of positive elements of $V(\mathfrak{B})$ and $B(\mathfrak{B})$ the subset of $K(\mathfrak{B})$ consisting of elements $\omega$ such that $\omega(e)=1 . K(\mathfrak{B})$ is the cone of positive normal linear functionals on $\mathfrak{B}$ and $B(\mathfrak{B})$ is the set of normal states of $\mathfrak{B}$. For $\omega \in K(\mathfrak{B})$, there exists $\left\{x_{n}\right\} \subset X$ such that

and

$$
\|\omega\|=\omega(e)=\sum_{n=1}^{\infty}\left\|x_{n}\right\|^{2}
$$

$$
\omega(T)=\sum_{n=1}^{\infty} \omega_{x_{n}}(T), \quad \forall T \in \mathfrak{B}
$$

where for $x \in X, \omega_{x}(T)=\langle T x, x\rangle, \forall T \in \mathfrak{B}$. Then, $(V(\mathfrak{B}), B(\mathfrak{B}))$ is a complete base norm space with norm closed cone $K(\mathfrak{B})$ and hence provides a suitable model for a statistical physical system. This is said to be the Von Neumann algebra model for quantum theory. The dual $\left(V^{*}(\mathfrak{B}), e\right)$ of $(V(\mathfrak{B}), B(\mathfrak{B}))$ is a complete order unit space, Archimedean ordered by the cone $K^{*}(\mathfrak{B}) . V^{*}(\mathfrak{B})$ and $K^{*}(\mathfrak{B})$ may be identified with the sets of self-adjoint and positive elements of $\mathfrak{B}$ respectively, and $e$ with the identity in $\mathfrak{B}$. Hence, the set $\mathscr{Q}$ of simple observables may be identified with the set of elements $A$ of $\mathfrak{B}$ such that $0 \leqq A \leqq e$. The set $E(2)$ of extreme points of $\mathscr{2}$ may be identified, therefore, with the set of projections in $\mathfrak{B}[16]$. It was shown in [7] that there is a one-one correspondence between restrictions of the system and central projections in $\mathfrak{B}$. In fact, for any central projection $A$, the set of states of the corresponding restriction is the set $\{\omega: \omega \in K(B), \omega(T)=\omega(A T A), \forall T \in \mathfrak{B}\}$ whilst the set of simple observables in the restriction is the set $\{T: T \in \mathfrak{B}, 0 \leqq T \leqq A\}$.

It is clear from the results of $\S 3$ that the norm closed extremal sets in $K(\mathfrak{B})$ play an important role in the theory. The following result, most of which is well known, summarizes their properties. For completeness a proof is given. First, some notation is established. For $\omega \in \mathfrak{B}_{*}, A, A^{\prime} \in \mathfrak{B}$, let $A \omega, \omega A^{\prime}, A \omega A^{\prime}$ be defined respectively by

$$
(A \omega)(T)=\omega(A T),\left(\omega A^{\prime}\right)(T)=\omega\left(T A^{\prime}\right),\left(A \omega A^{\prime}\right)(T)=\omega\left(A T A^{\prime}\right), \forall T \in \mathfrak{B}
$$

and for any subset $\mathfrak{L}$ of $\mathfrak{B}_{*}, A \mathfrak{L} A^{\prime}=\left\{A \omega A^{\prime}: \omega \in \mathfrak{L}\right\}$. 
Proposition 4.1.There exists a bijection $H \mapsto E_{H}$ between the set $\Pi(\mathfrak{B})$ of all norm closed extremal sets in $K(\mathfrak{B})$ and the set $E(2)$ of projections in $\mathfrak{B}$, defined by,

$E_{H}=$ minimal projection in $\mathfrak{B}$ such that $\omega=E_{H} \omega E_{H}, \forall \omega \in H$, $E_{H} X=\left\{x: x \in X, \omega_{x} \in H\right\}$,

$H=E_{H} K(\mathfrak{B}) E_{H}=\left\{\omega: \omega \in K(\mathfrak{B}), \omega\left(e-E_{H}\right)=0\right\}$.

$H \subset H^{\prime}$ if and only if $E_{H} \leqq E_{H^{\prime}}$ and, if $E_{H}, E_{H^{\prime}}$ are orthogonal, $H \cap H^{\prime}=\{0\}$. The mapping sends the set $\Gamma(\mathfrak{B})$ of all split extremal sets onto the set of central projections in $\mathfrak{B}$ and for $H \in \Gamma(\mathfrak{B})$ with complementary set $H^{\prime}, E_{H}+E_{H^{\prime}}=e$. If $H \in \Pi(\mathfrak{B})$, and $H^{\prime}$ is the smallest element of $\Gamma(B)$ containing $H, E_{H^{\prime}}$ is the central support of $E_{H}$.

Proof. For $H \in \Pi(\mathfrak{B})$, let $X_{H}=\left\{x: x \in X, \omega_{x} \in H\right\}$. For $x, y \in X_{H}, \alpha \in C$,

$$
\begin{aligned}
\omega_{x+y}+\omega_{x-y} & =2 \omega_{x}+2 \omega_{y} \in H, \\
\omega_{\alpha x} & =|\alpha|^{2} \omega_{x} \in H .
\end{aligned}
$$

Since $H$ is extremal, (4.3) shows that $x+y \in X_{H}$ and (4.4) shows that $\alpha x \in X_{H}$. Hence, $X_{H}$ is a subspace of $X$. If $\left\{x_{n}\right\} \subset X_{H}, x \in X$ and $\left\|x_{n}-x\right\| \rightarrow 0$ a simple limit argument shows that $\left\|\omega_{x_{n}}-\omega_{x}\right\| \rightarrow 0$ which implies, since $H$ is norm closed that $X_{H}$ is closed. Let $E_{H}$ be the corresponding projection onto $X_{H}$, For $U$ unitary in $\mathfrak{B}^{\prime}, \omega_{x}=\omega_{U x}, \forall x \in X$ and hence, $x \in X_{H}$ implies $U x \in X_{H}, \forall U$ unitary in $\mathfrak{B}^{\prime}$. It follows that $U E_{H}=E_{H} U, \forall U$ unitary in $\mathfrak{B}^{\prime}$ and, since $\mathfrak{B}^{\prime}$ is generated by its unitary elements, that $E_{H} \in \mathfrak{B}^{\prime \prime}=\mathfrak{B}$. For $\omega \in H$, let $\left\{x_{n}\right\} \subset X$ be such that (4.1), (4.2) hold. Then, since $H$ is extremal, $\omega_{x_{n}} \in H, \forall n$, which implies that $x_{n} \in E_{H} X, \forall n$. Since for $A \in \mathfrak{E}(X), x \in X, \omega_{A x}=A^{*} \omega_{x} A$, it follows that $\omega_{x_{n}}=\omega_{E_{H} x_{n}}=E_{H} \omega_{x_{n}} E_{H}$. A simple limit argument now shows that $\omega=E_{H} \omega E_{H}$. Suppose $P$ is a further projection in $\mathfrak{B}$ such that $\omega=P \omega P, \forall \omega \in H$. Then, for $x \in X_{H}$, $\omega_{x}=\omega_{P x}$ which implies that $\langle x, x\rangle=\langle P x, x\rangle$ and hence that $P x=x$. It follows that $E_{H} \leqq P$. Therefore the mapping $H \mapsto E_{H}$ sends $\Pi(\mathfrak{B})$ into $E(2)$.

Let $P \in E(\mathscr{Q})$ and let $H=K(\mathfrak{B}) \cap\{e-P\}_{0}$. Then $H$ is a norm closed extremal set and

$$
E_{H} X=\left\{x: x \in X, \omega_{x} \in H\right\}=\{x: x \in X, P x=x\}=P X .
$$

Therefore $E_{H}=P$ and $H \mapsto E_{H}$ maps onto $E(2)$. Let $H, H^{\prime} \in \Pi(B)$ and let $E_{H}=E_{H^{\prime}}$. Suppose $\omega \in H$ is given by (4.2). Then, $\omega_{x_{n}} \in H, \forall n$ and therefore, since $E_{H} X=E_{H^{\prime}} X, \omega_{x_{n}} \in H^{\prime}, \forall n$. Since $H^{\prime}$ is norm closed, $\omega \in H^{\prime}$ and therefore $H \subset H^{\prime}$. Similarly, $H^{\prime} \subset H$ and therefore $H \mapsto E_{H}$ is a bijection. Let $G=E_{H} K(\mathfrak{B}) E_{H}$. Then, for $\omega \in G, E_{H} \omega E_{H}=\omega$ which implies that $\omega\left(E_{H}\right)=\omega(e)$ and hence that $\omega \in H$. Therefore $G \subset H$ and conversely, for $\omega \in H, \omega=E_{H} \omega E_{H} \in G$. It follows that $G=H$ and the first part of the proof is complete. 
Let $H, H^{\prime} \in \Pi(\mathfrak{B})$ and let $H \subset H^{\prime}$. Then, clearly $X_{H} \subset X_{H^{\prime}}$ and therefore $E_{H} \leqq E_{H^{\prime}}$. Conversely, if $E_{H} \leqq E_{H^{\prime}}, \omega\left(E_{H}\right) \leqq \omega\left(E_{H^{\prime}}\right), \forall \omega \in K(\mathfrak{B})$. If $\omega \in H, \omega\left(e-E_{H}\right)=0$ and hence,

$$
0 \leqq \omega\left(e-E_{H^{\prime}}\right) \leqq \omega\left(e-E_{H}\right)=0
$$

which implies that $\omega \in H^{\prime}$. Hence $H \subset H^{\prime}$.

Suppose $H, H^{\prime} \in \Pi(\mathfrak{B}), E_{H} E_{H^{\prime}}=0, \omega \in H \cap H^{\prime}$. Then,

$$
\omega=E_{H} \omega E_{H}=E_{H^{\prime}} E_{H} \omega E_{H} E_{H^{\prime}}=0 .
$$

Next, suppose that $E_{H}$ is central and let $\omega \in K(\mathfrak{B})$. Then,

$$
\omega=E_{H} \omega E_{H}+\left(e-E_{H}\right) \omega\left(e-E_{H}\right)
$$

where $E_{H} \omega E_{H} \in H,\left(e-E_{H}\right) \omega\left(e-E_{H}\right) \in \tilde{H}=\left(e-E_{H}\right) K(\mathfrak{B})\left(e-E_{H}\right) \in \Pi(\mathfrak{B})$. Suppose, alternatively that $\omega=\omega_{1}+\omega_{2}, \omega_{1} \in H, \omega_{2} \in \tilde{H}$. Then,

and

$$
E_{H} \omega_{1} E_{H}=\omega_{1},\left(e-E_{H}\right) \omega_{2}\left(e-E_{H}\right)=\omega_{2},
$$

$$
E_{H} \omega E_{H}=E_{H} \omega_{1} E_{H}+E_{H} \omega_{2} E_{H}=E_{H} \omega_{1} E_{H}=\omega_{1} .
$$

Similarly, $\left(e-E_{H}\right) \omega\left(e-E_{H}\right)=\omega_{2}$ and the decomposition (4.5) is unique. Suppose $G \in \Pi(\mathfrak{B}), H \cap G=\{0\}$. Then, since $E_{H} E_{G}=E_{G} E_{H}$, it follows that $E_{H} E_{G}$ is the projection onto $X_{H} \cap X_{G}$. If $E_{H} E_{G} \neq 0$, there exists $x \in X_{H} \cap X_{G}, x \neq 0$ and therefore $\omega_{x} \in H \cap G$ giving a contradiction. Therefore, $E_{H}, E_{G}$ are orthogonal projections and $E_{G} \leqq e-E_{H}$. It follows that $G \subset \tilde{H}$, and therefore that the complementary set $H^{\prime}$ of $H$ is contained in $\tilde{H}$. However, $\tilde{H} \in \Pi(\mathfrak{B}), H \cap \tilde{H}=\{0\}$ and therefore $\tilde{H} \subset H^{\prime}$. Hence, $\tilde{H}=H^{\prime}$ and $H$ is a split extremal set. Conversely, let $H$ be a split extremal set. Then, it follows from Prop. 3.12 and Theorem 6.1 of [7] that $E_{H}$ is central. Hence, $H \mapsto E_{H}$ is a bijection between $\Gamma(\mathfrak{B})$ and the set of central projections in $\mathfrak{B}$.

Finally, let $H \in \Pi(\mathfrak{B})$ and let $G$ be the smallest element of $\Gamma(\mathfrak{B})$ containing $H$. Then, $E_{H} \leqq E_{G}$ and, if $E_{G^{*}}$ is any central projection such that $E_{H} \leqq E_{G^{n}}, H \subset G^{\wedge}$ which implies that $G \subset G^{\wedge}, E_{G} \leqq E_{G^{\wedge}}$. Therefore $E_{G}$ is the central support of $E_{H}$. The converse assertion is proved by reversing this argument. This completes the proof.

It is now possible to characterize certain of the classes of operations discussed in $\S 3$. For $A \in \mathcal{Q}$, recall that the sets $H_{A}, K_{A}$ of transmitted and non-transmitted states respectively, are norm closed extremal sets in $K(\mathfrak{B})$ and hence there exist unique projections $P_{A}=E_{H_{A}}, Q_{A}=E_{K_{A}}$ in $\mathfrak{B}$ such that $H_{A}=P_{A} K(\mathfrak{B}) P_{A}, K_{A}=Q_{A} K(\mathfrak{B}) Q_{A}$. Prop. 4.1 shows that $x \in P_{A} X$ if and only if $\omega_{x}(e-A)=0$ and therefore if and only if $x$ lies in the null space of $e-A$. It follows that $P_{A}$ is the projection onto the null space of $e-A$ and therefore that $e-P_{A}$ is the support of $e-A$. Similarly, 
$e-Q_{A}$ is the support of $A$. Further, for $x \in Q_{A} X, A x=0$ and $(e-A) x=x$ which implies that $x$ lies in the range of $e-A$. The range projection of $e-A$ is precisely the support of $e-A$ and hence $Q_{A} \leqq e-P_{A}$ and $Q_{A}, P_{A}$ are orthogonal projections.

In particular, for $A \in E(2), P_{A}=A, Q_{A}=e-A$. Conversely, if $P_{A}+Q_{A}$ $=e$, the range projection of $e-A$ coincides with the null projection of $A$ and therefore $A(e-A) x=0, \forall x \in X, A=A^{2}$ and $A \in E(2)$. The properties of simple observables in the Von Neumann algebra model are given below.

Proposition 4.2. The set 2 of simple observables is the set of positive elements $A$ of $\mathfrak{B}$ satisfying $A \leqq e$ and the set $E(2)$ of extreme simple observables is the set of projections in $\mathfrak{B}$. For $A \in \mathscr{Q}$, there exist uniquely, orthogonal projections $P_{A}, Q_{A}$ in $\mathfrak{B}$ such that

$$
H_{A}=P_{A} K(\mathfrak{B}) P_{A}, \quad K_{A}=Q_{A} K(\mathfrak{B}) Q_{A}
$$

and $e-P_{A}, e-Q_{A}$ are the supports of $e-A, A$ respectively. $P_{A}+Q_{A}=e$ if and only if $A \in E(2)$ in which case $P_{A}=A, Q_{A}=e-A$.

Notice that the Davies-Lewis definition of material implication, discussed in $\S 3$, leads to the conclusion in this example that, for $A_{1}, A_{2} \in \mathscr{Q}$, $A_{1} \Rightarrow A_{2}$ if and only $P_{A_{1}} \leqq P_{A_{2}}$, where $e-P_{A_{1}}, e-P_{A_{2}}$ are the range projections of $e-A_{1}, e-A_{2}$. For elements of $E(\mathscr{Q})$ this definition coincides with Mackey's definition, namely that $A_{1} \Rightarrow A_{2}$ if and only if $A_{1} \leqq A_{2}$.

Let $\mathscr{P}$ be the set of operations on the system. Then, $j \in \mathscr{P}$ is a positive linear mapping from $V(\mathfrak{B})$ to itself such that $\|j\| \leqq 1$ and clearly extends to a positive linear mapping from $\mathfrak{B}_{*}=V(\mathfrak{B})+i V(\mathfrak{B})$ to itself. The adjoint $j^{*}$ of $j$ is an ultraweakly continuous positive linear mapping from $\mathfrak{B}$ to itself. Lemma 5.1 of [20] shows that there exists a minimal projection $E_{j}$ in $\mathfrak{B}$ such that $j^{*}(T)=j^{*}\left(E_{j} T E_{j}\right), \forall T \in \mathfrak{B}$ and $E_{j} T E_{j} \mapsto j^{*}\left(E_{j} T E_{j}\right)$ is faithful. It follows that $E_{j}$ is the minimal projection in $\mathfrak{B}$ such that $E_{j} \omega E_{j}=\omega, \forall \omega \in j(K(\mathfrak{B}))$. Hence, $N_{j}=E_{j} K(\mathfrak{B}) E_{j}$ is the smallest element of $\Pi(\mathfrak{B})$ containing $j(K(\mathfrak{B}))$.

Recall that the mapping $j \mapsto T(j)$ from $\mathscr{P}$ onto $\mathscr{Q}$ is defined by $\omega(T(j))=j(\omega)(e), \forall \omega \in K(\mathfrak{B})$ or alternatively by $T(j)=j^{*}(e)$. Generally, there are many elements $j$ of $\mathscr{P}$ such that $T(j)=A$ [20]. It was shown in $\S 3$ that, for $v \in B(\mathfrak{B})$,

$$
j(\omega)=\omega(A) v, \quad \forall \omega \in K(\mathfrak{B})
$$

is such an operation and clearly

$$
j(\omega)=A^{\frac{1}{2}} \omega A^{\frac{1}{2}}, \quad \forall \omega \in K(\mathfrak{B})
$$

is a further example. 
For $j \in \mathscr{P}$, let $j^{\prime} \in \mathscr{L}_{j}$. Then, $j(\omega)\left(T\left(j^{\prime}\right)\right)=0, \forall \omega \in K(\mathfrak{B})$ and hence $j(K(\mathfrak{B})) \subset K_{T\left(j^{\prime}\right)}$ which implies that $N_{j} \subset K_{T\left(j^{\prime}\right)}$ and therefore, using Prop. 4.1 , that $E_{j} \leqq Q_{T\left(j^{\prime}\right)}$. Conversely, if $E_{j} \leqq Q_{T\left(j^{\prime}\right)}, N_{j} \subset K_{T\left(j^{\prime}\right)}$ and $j(K(\mathfrak{B}))$ $\subset K_{T\left(j^{\prime}\right)}$ which implies that $j^{\prime} \in \mathscr{L}_{j}$.

Suppose $\mathscr{T}_{j} \cap \mathscr{L}_{j} \neq \emptyset$. Then, using Prop. 3.5 (iv), $\mathscr{T}_{j} \subset \mathscr{L}_{j}$ and hence for $j^{\prime} \in \mathscr{T}_{j}, E_{j} \leqq Q_{T\left(j^{\prime}\right)}=Q_{e-T(j)}=P_{T(j)}$. Conversely, if $E_{j} \leqq P_{T(j)}=Q_{T\left(j^{\prime}\right)}$, then, from above $j^{\prime} \in \mathscr{L}_{j}$. Summarizing,

Proposition 4.3. (i) For $j \in \mathscr{P}, j^{\prime} \in \mathscr{L}_{j}$ if and only if $E_{j} \leqq Q_{T\left(j^{\prime}\right)}$ and $j, j^{\prime}$ are disjoint if and only if $E_{j} \leqq Q_{T\left(j^{\prime}\right)}, E_{j^{\prime}} \leqq Q_{T(j)}$.

(ii) For $j \in \mathscr{P}, \mathscr{T}_{j} \cap \mathscr{L}_{j} \neq \emptyset$ if and only if $E_{j} \leqq P_{T(j)}$ and $\mathscr{T}_{j} \cap \mathscr{U}_{j} \neq \emptyset$ if and only if $E_{j} \leqq P_{T(j)}$ and there exists $j^{\prime} \in \mathscr{T}_{j}$ such that $E_{j^{\prime}} \leqq P_{T\left(j^{\prime}\right)}$.

It follows from Prop. 4.3 that $j \in \mathscr{P}$ is repeatable if and only if there exists $j^{\prime} \in \mathscr{P}$ such that,

(i) $T(j)+T\left(j^{\prime}\right)=e$,

(ii) $E_{j} \leqq P_{T(j)}, E_{j^{\prime}} \leqq P_{T\left(j^{\prime}\right)}$.

Since $P_{T(j)}, P_{T\left(j^{\prime}\right)}$ are orthogonal, it follows from (ii) that $E_{j}, E_{j^{\prime}}$ are orthogonal. Further, $j$ is (a) or (c)-repeatable according as,

(a) $A \in \mathscr{Q}, E_{j} \leqq P_{A}, E_{j^{\prime}} \leqq P_{e-A}$ implies $A=T(j)$,

(c) $A \in \mathscr{Q}, E_{j} \leqq Q_{A}, E_{j^{\prime}} \leqq Q_{A}$ implies $A=0$, respectively.

Suppose first that $j$ is (a)-repeatable with complementary repeatable operation $j^{\prime}$. Then, $E_{j}+E_{j^{\prime}} \leqq e$, and applying (a) above with $A=E_{j}$ and $A=e-E_{j^{\prime}}$ respectively gives $E_{j}=T(j), e-E_{j^{\prime}}=T(j)$. Hence $E_{j}+E_{j^{\prime}}=e$.

Conversely, suppose $j$ is repeatable with complementary repeatable operation $j^{\prime}$ such that $E_{j}+E_{j^{\prime}}=e$. Let $A \in \mathscr{Q}$ satisfy $E_{j} \leqq P_{A}, E_{j^{\prime}} \leqq P_{e-A}$. Then, $e=E_{j}+E_{j^{\prime}} \leqq P_{A}+P_{e-A}=P_{A}+Q_{A}$. Therefore $P_{A}+Q_{A}=e$ and Prop. 4.2 shows that $P_{A}=A, Q_{A}=e-A$. It follows then that $E_{j} \leqq A$, $e-E_{j} \leqq P_{e-A}=e-A$ and hence $E_{j}=A, E_{j^{\prime}}=e-A$. In particular, this holds when $A=T(j)$. It follows that $E_{j}=T(j), E_{j^{\prime}}=T\left(j^{\prime}\right)$ and $j$ is (a)repeatable.

Next, suppose that $j$ is (c)-repeatable with complementary repeatable operation $j^{\prime}$. Then, $E_{j}+E_{j^{\prime}} \leqq e$ and applying (c) above with $A=e-E_{j}$ $-E_{j^{\prime}}$, gives $E_{j}+E_{j^{\prime}}=e$.

Conversely, let $j$ be repeatable with complementary repeatable operation $j^{\prime}$ such that $E_{j}+E_{j^{\prime}}=e$. Then, as before $E_{j}=T(j), E_{j^{\prime}}=T\left(j^{\prime}\right)$, and if $A \in \mathscr{Q}$ satisfies $E_{j} \leqq Q_{A}, E_{j^{\prime}} \leqq Q_{A}$, since $E_{j}, E_{j^{\prime}}$ are orthogonal, $e=E_{j}+E_{j^{\prime}}$ $\leqq Q_{A}$. It follows that $Q_{A}=e$ and therefore $A=0$.

The following result has been proved.

Proposition 4.4. (i) $j \in \mathscr{P}$ is repeatable with complementary repeatable operation $j^{\prime}$ if and only if $E_{j} \leqq P_{T(j)}, E_{j^{\prime}} \leqq P_{T\left(j^{\prime}\right)}$. 
(ii) The following conditions on a repeatable operation $j$ with complementary repeatable operation $j^{\prime}$ are equivalent,

(a) $j$ is (a)-repeatable,

(b) $E_{j}+E_{j^{\prime}}=e$,

(c) $j$ is (c)-repeatable, and if any of these conditions hold $E_{j}=T(j), E_{j^{\prime}}=T\left(j^{\prime}\right)$.

For $j \in \mathscr{P}, G_{j}=\{\omega: \omega \in K(\mathfrak{B}), j(\omega)=\omega\}$ is a norm closed convex subset of $K(\mathfrak{B})$ such that $G_{j} \subset j(K(\mathfrak{B}))$. Let $M_{j}$ be the smallest element of $\Pi(\mathfrak{B})$ containing $G_{j}$ and let $F_{j}=E_{M_{j}}$. Then, $M_{j} \subset N_{j}, F_{j} \leqq E_{j}$ and $F_{j}$ is the minimal projection in $\mathfrak{B}$ such that

$$
j\left(F_{j} \omega F_{j}\right)=F_{j} \omega F_{j}, \quad \forall \omega \in K(\mathfrak{B}) .
$$

$j$ is a filtering if and only if $G_{j}=H_{T(j)}$ and hence, $j \in \mathscr{P}_{F}$ implies $F_{j}=P_{T(j)}$.

Let $j$ be a repeatable operation with complementary repeatable operation $j^{\prime}$, Then,

$$
\begin{gathered}
G_{j} \subset j(K(\mathfrak{B})) \subset H_{T(j)}, \quad G_{j^{\prime}} \subset j^{\prime}(K(\mathfrak{B})) \subset H_{T\left(j^{\prime}\right)}, \\
M_{j} \subset N_{j} \subset H_{T(j)}, \quad M_{j^{\prime}} \subset N_{j^{\prime}} \subset H_{T\left(j^{\prime}\right)}, \quad F_{j} \leqq E_{j} \leqq P_{T(j)}, \quad F_{j^{\prime}} \leqq E_{j^{\prime}} \leqq P_{T\left(j^{\prime}\right)}
\end{gathered}
$$

and if $j, j^{\prime} \in \mathscr{P}_{F}$,

$$
\begin{gathered}
G_{j}=M_{j}=j(K(\mathfrak{B}))=N_{j}=H_{T(j)}, \quad G_{j^{\prime}}=M_{j^{\prime}}=j^{\prime}(K(\mathfrak{B}))=N_{j^{\prime}}=H_{T\left(j^{\prime}\right)}, \\
F_{j}=E_{j}=P_{T(j)}, \quad F_{j^{\prime}}=E_{j^{\prime}}=P_{T\left(j^{\prime}\right)} .
\end{gathered}
$$

It follows that $j$ is strongly $(a)$-repeatable if and only if $F_{j}+F_{j^{\prime}}=e$.

Proposition 4.5. Let $j \in \mathscr{P}$ be repeatable with complementary repeatable operation $j^{\prime}$ and let $j, j^{\prime} \in \mathscr{P}_{F}$. Then $j$ is strongly (a) or (c)-repeatable if and only if $F_{j}+F_{j^{\prime}}=e$.

It follows from Prop. 3.11 and Prop. 4.1 that the set of strongly repeatable reflections can be characterized as follows.

Proposition 4.6. There exists a one-one correspondence between elements $j$ of $\mathscr{P}_{\mathrm{SR} W}$ and central projections $T(j)$ in $\mathfrak{B}$ defined by

$$
j(\omega)=T(j) \omega T(j), \quad \forall \omega \in K(\mathfrak{B}) .
$$

It follows from Prop. 3.12 that every strong restriction of the system is also a restriction. An immediate corollary of Prop. 4.6 is the following.

Proposition 4.7. In the Von Neumann algebra model, every restriction is a strong restriction.

4 Commun. math. Phys., Vol. 20 


\section{§ 5. "Pure" Operations in the Von Neumann Algebra Model}

As was remarked above, for $A \in \mathscr{Q}$, there are two obvious types of operation $j$ such that $T(j)=A$, defined by

$$
\begin{aligned}
\text { I } j(\omega)=\omega(A) v, \quad v \in B(\mathfrak{B}), \quad \forall \omega \in K(\mathfrak{B}), \\
\text { II } j(\omega)=A^{\frac{1}{2}} \omega A^{\frac{1}{2}}, \quad \forall \omega \in K(\mathfrak{B}) .
\end{aligned}
$$

This second class can be widened considerably, for if $U$ is a bounded linear or anti-linear operator on $X$ such that,

(i) $U^{*} U=A$,

(ii) $U^{*} \mathfrak{B} U \subset \mathfrak{B}$,

then, if

$j \in \mathscr{P}, T(j)=A$.

$$
j(\omega)=U^{*} \omega U, \quad \forall \omega \in K(\mathfrak{B}),
$$

It is shown in [3] that when $X$ is separable and $\mathfrak{B}=\mathfrak{Q}(X)$, every operation mapping the set $\mathscr{D}$ of extremal sets in $K(\mathfrak{B})$ of the form $\{\alpha v: v \in B(\mathfrak{B}), \alpha \geqq 0\}$ into itself is of one of the kinds described above. Further discussion of these kinds of operation has been given by Haag and Kastler [13] and Hellwig and Kraus [14, 15]. In both cases such operations were said to be "pure" operations. For an arbitrary Von Neumann algebra $\mathfrak{B}$, operations of the first kind map $\mathscr{D}$ into $\mathscr{D}$ providing $v$ is chosen to be an extreme point of $B(\mathfrak{B})$ though the same is not necessarily true for operations of the second kind. It is, of course, true when $\mathfrak{B}=\mathfrak{L}(X)$. All that can be said about operations of the second kind is that they map vector states into vector states and clearly every extreme point of $B(\mathfrak{B})$ is a vector state. The most that can be said about operations of the first kind is that they map $\mathscr{D}$ into $\mathscr{D}$ provided that $v \in E(B(\mathfrak{B}))$.

The structure of $\mathscr{D}$ is intimately associated with the Type of $\mathfrak{B}$ and it may be that $\mathscr{D}$ contains very few elements. However, when $\mathfrak{B}$ is the Von Neumann envelope of a $C^{*}$-algebra $\mathfrak{A}, \mathscr{D}$ is isomorphic to the set of pure states of $\mathfrak{A}$ and $B(\mathfrak{B})$ is the closed convex hull of its set of extreme points.

\section{Let}

$$
\begin{aligned}
\mathscr{P}^{\mathrm{I}}= & \{j: j \in \mathscr{P}, \exists A \in \mathscr{Q}, v \in B(\mathfrak{B}), j(\omega)=\omega(A) v, \forall \omega \in K(\mathfrak{B})\}, \\
\mathscr{P}^{\mathrm{II}}= & \left\{j: j \in \mathscr{P}, \exists U \in \mathfrak{Q}(X),\|U\| \leqq 1, U^{*} \mathfrak{B} U \subset \mathfrak{B}, j(\omega)=U^{*} \omega U,\right. \\
& \forall \omega \in K(\mathfrak{B})\} .
\end{aligned}
$$

The properties of these classes of operations are now discussed in the light of $\S \S 3,4$.

Let $j \in \mathscr{P}^{\mathrm{I}}$ be defined by

$$
j(\omega)=\omega(A) v, A \in \mathscr{Q}, A \neq 0, v \in B(\mathfrak{B}), \forall \omega \in K(\mathfrak{B}) .
$$

Then $N_{j}$ is the smallest element of $\Pi(\mathfrak{B})$ containing $v$ and therefore, $E_{j}=E_{v}$, the support of $v$. In particular, if $v$ is an extreme point of $B(\mathfrak{B})$, 
that is, a pure normal state, then $N_{j}=\{\alpha v: \alpha \geqq 0\}$. Further,

$$
G_{j}=\{\omega: \omega \in K(\mathfrak{B}), \omega(A) v=\omega\}
$$

and if $G_{j} \neq\{0\}, N_{j}=M_{j}, E_{j}=F_{j}$. Notice that $G_{j} \neq\{0\}$ provided that $v(A) \neq 0$, for in this case $v / v(A) \in G_{j}$.

$j \in \mathscr{P}_{T}$ if and only if $j(\omega)=\omega(e) v, \forall \omega \in K(\mathfrak{B})$ and $j \in \mathscr{P}_{S}$ if and only if $K(\mathfrak{B})=\{\alpha v: \alpha \geqq 0\}$ and therefore, if and only if $\mathfrak{B}=\boldsymbol{C}$.

Let $j^{\prime} \in \mathscr{P}^{\mathrm{I}}$ be defined by

$$
j^{\prime}(\omega)=\omega\left(A^{\prime}\right) v^{\prime}, A^{\prime} \in \mathscr{Q}, A^{\prime} \neq 0, v^{\prime} \in B(\mathfrak{B}), \forall \omega \in K(\mathfrak{B}) .
$$

Then, $j j^{\prime}(\omega)=\omega\left(v^{\prime}(A) A^{\prime}\right) v$ and it follows that $j j^{\prime} \in \mathscr{P}^{\mathrm{I}}$ and $T\left(j j^{\prime}\right)=v^{\prime}(A) A^{\prime}$. Therefore, $j, j^{\prime}$ commute weakly if and only if $v^{\prime}(A) A^{\prime}=v\left(A^{\prime}\right) A$ and commute strongly if and only if in addition, $v=v^{\prime}$. In particular, $j, j^{\prime}$ are disjoint if and only if $v^{\prime}(A)=v\left(A^{\prime}\right)=0$. Clearly,

$$
j^{\prime}(\omega)=\omega(e-A) v^{\prime}, v^{\prime} \in B(\mathfrak{B}), \forall \omega \in K(\mathfrak{B}),
$$

defines an element of $\mathscr{P}^{\mathrm{I}} \cap \mathscr{T}_{j}$ and every element of $\mathscr{P}^{\mathrm{I}} \cap \mathscr{T}_{j}$ is of this form. It follows that $j$ is repeatable with complementary repeatable operation $j^{\prime}$ given (5.3) if and only if $v^{\prime}(A)=0, v(A)=1$, or, equivalently, if and only if $E_{v} \leqq P_{A}, E_{v^{\prime}} \leqq P_{e-A}$. Further, it follows from Prop. 4.4 that $j$ is (a) (or (c))-repeatable if and only if $E_{v}=A, E_{v^{\prime}}=e-A$, in which case $A, e-A$ are the supports of $v, v^{\prime}$ respectively.

Next, suppose that $j$, defined by (5.1), is an element of $\mathscr{P}_{F}$ such that $G_{j} \neq\{0\}$. Then, $H_{A}=G_{j} \subset\{\alpha v: \alpha \geqq 0\}$ and hence, $H_{A}=G_{j}=\{\alpha v: \alpha \geqq 0\}$. Since $v \in G_{j}$, it follows that $v(A)=1$ and that $v$ is a pure normal state with support $E_{v}=P_{A}$. Conversely, suppose that $v$ is a pure normal state with support $P_{A}$. It follows that $H_{A}=\{\alpha v: \alpha \geqq 0\}$ and since $v \in H_{A}$, that $v(A)=1$. Therefore $v \in G_{j}$ and $H_{A}=\{\alpha v: \alpha \geqq 0\} \subset G_{j}$. But $G_{j} \subset H_{A}$ and so it follows that $H_{A}=G_{j} \neq\{0\}$.

It follows from the two results above that $j$, defined by (5.1) is strongly (a) (or (c))-repeatable with complementary repeatable operation $j^{\prime}$ defined by (5.3) if and only if $v, v^{\prime}$ are pure normal states with supports $A, e-A$ respectively.

Suppose $j$, defined by (5.1) is a strong operation. Then, Prop. 4.6 shows that for each central projection $P$ in $\mathfrak{B}$,

$$
(P \omega P)(A) v=j(P \omega P)=P j(\omega) P=\omega(A) P v P, \quad \forall \omega \in K(\mathfrak{B})
$$

and, replacing $\omega$ by $P \omega P$, that $\omega(P A P)=\omega(P A P) v(P), \forall \omega \in K(\mathfrak{B})$. Therefore, either $P A P=0$ or $v(P)=1$. If $P A P=0, A=(e-P) A(e-P)$. Since $e-P$ is a central projection it follows that $v(e-P)=1$, $v(P)=0$. If $v(P)=1$, then $v(e-P)=0$ and so $(e-P) A(e-P)=0$, $A=P A P$. Therefore, for each central projection $P$, either $P A P=0$, $v(P)=0$, or $P A P=A, v(P)=1$. Let $C_{A}$ be the central support of $A$ and 
suppose $P$ is a central projection such that $P \leqq C_{A}, P \neq C_{A}$. Then, $P A P \neq A$ and therefore, $P A P=0$ which implies that $P=P C_{A}=0$. Therefore $C_{A}$ is a minimal central projection in $\mathfrak{B}$ such that $v\left(C_{A}\right)=1$.

Conversely, let $C_{A}$ be a minimal central projection in $\mathfrak{B}$ such that $v\left(C_{A}\right)=1$, and let $P$ be any central projection in $\mathfrak{B}$. Then, either $P C_{A}=C_{A} P$ $=0$ or $P C_{A}=C_{A} P=C_{A}$ and either, $P A P=P C_{A} A C_{A} P=0$ or $P A P$ $=P C_{A} A C_{A} P=C_{A} A C_{A}=A$. In the first case,

$$
v(P)=v\left(C_{A} P\right)+v\left(\left(e-C_{A}\right) P\right) \leqq v\left(e-C_{A}\right)=0
$$

and $v(P)=0$. In the second case,

$$
v(e-P)=v\left(C_{A}(e-P)\right)+v\left(\left(e-C_{A}\right)(e-P)\right) \leqq v\left(e-C_{A}\right)=0
$$

and $v(P)=1$. If $P A P=0$ and $v(P)=0$,

$$
j(P \omega P)=\omega(P A P) v=0, \quad P j(\omega) P=\omega(A) P v P=0, \quad \forall \omega \in K(\mathfrak{B}),
$$

since $v(P)=0$ and using Prop. 4.1. If $P A P=A$ and $v(P)=1$.

$$
j(P \omega P)=\omega(A) v, \quad P_{j}(\omega) P=\omega(A) P v P=\omega(A) v, \quad \forall \omega \in K(B),
$$

since $v(e-P)=0$ and using Prop. 4.1. Therefore, for each central projection $P$ in $\mathfrak{B}, P j(\omega) P=j(P \omega P)$ and Prop. 4.6 shows that $j$ is a strong operation.

The properties of $\mathscr{P}$ I are listed below.

Proposition 5.1. Let $j, j^{\prime} \in \mathscr{P}^{\mathrm{l}}$ be defined by (5.1), (5.3) respectively. Then,

(i) $E_{j}=E_{v}$ the support of $v$ and if $G_{j} \neq\{0\}, E_{j}=F_{j}$.

(ii) $j \in \mathscr{P}_{S}$ if and only if $A=e$ and $\mathfrak{B}=\boldsymbol{C}$.

(iii) $j \in \mathscr{P}_{R}$ with complementary repeatable operation $j^{\prime}$ if and only if $E_{v} \leqq P_{A}, E_{v} \leqq P_{e-A}$.

(iv) $j \in \mathscr{P}_{R}$ is (a) (or (c))-repeatable with complementary repeatable operation $j^{\prime}$ if and only if $E_{v}=A, E_{v^{\prime}}=e-A$.

(v) $j \in \mathscr{P}_{F}, G_{j} \neq\{0\}$, if and only if $v$ is a pure normal state with support $P_{A}$.

(vi) $j \in \mathscr{P}_{S R a}\left(\right.$ or $\mathscr{P}_{S R c}$ ) with complementary repeatable operation $j^{\prime}$ if and only if $v, v^{\prime}$ are pure normal states with supports $A, e-A$ respectively.

(vii) $j$ is a strong operation if and only if the central support $C_{A}$ of $A$ is a minimal central projection such that $v\left(C_{A}\right)=1$.

Let $j \in \mathscr{P}^{\mathrm{Il}}$ be defined by

$$
j(\omega)=U^{*} \omega U, U \in \mathfrak{I}(X),\|U\| \leqq 1, U^{*} \mathfrak{B} U \subset \mathfrak{B}, \quad \forall \omega \in K(\mathfrak{B}), \quad \text { (5.4) }
$$

and let $A=T(j)=U^{*} U$. Then, if $S_{U}, S_{U^{*}}$ are the supports of $U, U^{*}$ respectively then $S_{U}, S_{U^{*}}$ are the range projections of $U^{*}, U$ respectively and if $U \in \mathfrak{B}, U^{*}, S_{U}, S_{U^{*}} \in \mathfrak{B}$. Since $Q_{A}$ is the null projection of $U^{*} U$, it 
follows that $Q_{A}=e-S_{U} . A \in E(2)$ if and only if $U$ is a partial isometry with initial projection $A=U^{*} U=S_{U}$ and in this case the final projection of $U$ is $U U^{*}=S_{U^{*}}$. For $x \in X, j\left(\omega_{x}\right)=\omega_{U x}$ and it follows that $E_{j}$ is the minimal projection in $\mathfrak{B}$ such that $S_{U^{*}} \leqq E_{j}$. In particular, for $U \in \mathfrak{B}, S_{U^{*}}=E_{j}$.

$j \in \mathscr{P}_{T}$ if and only if $U^{*} U=e$ and therefore if and only if $U$ is a partial isometry with initial projection $e . j \in \mathscr{P}_{S}$ if and only if in addition $j(K(\mathfrak{B}))=K(\mathfrak{B})$ and hence, if and only if $U$ is unitary.

Let $j^{\prime} \in \mathscr{P}^{\text {II }}$ be defined by

$$
j^{\prime}(\omega)=U^{*} \omega U^{\prime}, U^{\prime} \in \mathbb{P}(X),\left\|U^{\prime}\right\| \leqq 1, U^{\prime} * \mathfrak{B} U^{\prime} \subset \mathfrak{B}, \forall \omega \in K(B),
$$

and if $A^{\prime}=T\left(j^{\prime}\right)=U^{\prime *} U^{\prime}$, suppose $A^{\prime} \neq 0$. If $j$ is defined by (5.4), and $A \neq 0$, then

$$
j j^{\prime}(\omega)=\left(U^{\prime} U\right)^{*} \omega\left(U^{\prime} U\right), \forall \omega \in K(\mathfrak{B})
$$

and $j j^{\prime} \in \mathscr{P}^{\text {II }}, T\left(j j^{\prime}\right)=\left(U^{\prime} U\right)^{*}\left(U^{\prime} U\right)$. It follows that $j, j^{\prime}$ are disjoint if and only if $U^{\prime} U=U U^{\prime}=0$ or equivalently, if and only if

Clearly,

$$
S_{U^{*}} \leqq e-S_{U^{\prime}}=Q_{A^{\prime}}, S_{U^{*}} \leqq e-S_{U}=Q_{A} .
$$

$$
j^{\prime \prime}(\omega)=(e-A)^{\frac{1}{2}} \omega(e-A)^{\frac{1}{2}}, \forall \omega \in K(B),
$$

defines an element of $\mathscr{P P I}^{\mathrm{II}} \cap \mathscr{T}_{j}$ which is therefore non-empty.

Let $j$ be defined by (5.4) and let $j^{\prime}$ be defined by (5.5), with $A^{\prime}=e-A$. Then, it follows from (5.7) that $j$ is repeatable with complementary repeatable operation $j^{\prime}$ if and only if

$$
S_{U *} \leqq P_{A}, S_{U^{\prime} *} \leqq P_{e-A} .
$$

Now suppose that $j$ is (a) (or (c))-repeatable. Then, $E_{j}=A, E_{j^{\prime}}=e-A$ from Prop. 4.4, and $U, U^{\prime}$ are partial isometries with initial projections $A, e-A$ and final projections $S_{U^{*}}=U U^{*}, S_{U^{*}}=U^{\prime} U^{\prime *}$ respectively. From (5.8), it follows that

$$
S_{U^{*}} \leqq A, S_{U^{*}} \leqq e-A .
$$

Conversely, let $j, j^{\prime}$ be defined by (5.4), (5.5) respectively with $A^{\prime}=e-A$, $U, U^{\prime}$ partial isometries and suppose $E_{j}+E_{j^{\prime}}=e, U U^{*} \leqq A, U^{\prime} U^{\prime *} \leqq e$ $-A$. Then, from above $j$ is repeatable with complementary repeatable operation $j^{\prime}$ and, in addition, Prop. 4.4 shows that $j$ is (a) (and (c))repeatable.

When $U, U^{\prime} \in \mathfrak{B}$, and $j$ is (a) (or (c))-repeatable with complementary repeatable operation $j^{\prime}$, using the fact that $S_{U^{*}}=E_{j}, S_{U^{\prime *}}=E_{j^{\prime}}$, it follows that

$$
U U^{*}=S_{U^{*}}=E_{j}=A=U^{*} U, U^{\prime} U^{*}=S_{U^{*}}=E_{j^{\prime}}=e-A=U^{\prime *} U^{\prime} .
$$


Conversely, when $U U^{\prime} \in \mathfrak{B}$, are partial isometries such that

$$
U^{*} U=U U^{*}, U^{*} U^{\prime}=U^{\prime} U^{\prime *}, U^{*} U+U^{\prime *} U^{\prime}=e,
$$

(5.4) defines an (a) (and (c))-repeatable operation $j$ with complementary repeatable operation $j^{\prime}$ defined by (5.5).

Let $j, j^{\prime}$ be defined by (5.4), (5.5) and suppose that $j$ is strongly (a) (or (c))-repeatable with complementary repeatable operation $j^{\prime}$. It follows from (5.9) that the ranges of $U, U^{\prime}$ are contained in $A X,(e-A) X$ respectively and hence that

For $\omega \in K(\mathfrak{B})$,

$$
\begin{aligned}
& A U=U,(e-A) U^{\prime}=U^{\prime}, \\
& U^{*} A=U^{*}, U^{\prime *}(e-A)=U^{\prime *} .
\end{aligned}
$$

$$
\omega=A \omega A+(e-A) \omega A+A \omega(e-A)+(e-A) \omega(e-A)
$$

and therefore, since $A \omega A \in H_{A}=G_{j},(e-A) \omega(e-A) \in K_{A}$,

$$
\begin{aligned}
& j(\omega)=A \omega A+j((e-A) \omega A+A \omega(e-A)) \\
& \quad=A \omega A+U^{*}(e-A) \omega A U+U^{*} A \omega(e-A) U
\end{aligned}
$$

where $j$ is extended to $\mathfrak{B}_{*}$ by linearity,

$$
\begin{aligned}
= & A \omega A+\left(U^{*}-U^{*} A\right) \omega A U+U^{*} A \omega(U-A U) \\
& =A \omega A,
\end{aligned}
$$

using (5.10), (5.11). Similarly,

$$
j^{\prime}(\omega)=(e-A) \omega(e-A) .
$$

Conversely, suppose that $j, j^{\prime}$ are defined by (5.4), (5.5) and

$$
j(\omega)=A \omega A, j^{\prime}(\omega)=A^{\prime} \omega A^{\prime}, \forall \omega \in K(\mathfrak{B})
$$

where $A^{\prime}=e-A$. Then,

$$
\omega\left(\left(U^{*} U\right)^{2}\right)=\omega\left(U^{*} U\right), \omega\left(\left(U^{*} U^{\prime}\right)^{2}\right)=\omega\left(U^{\prime *} U^{\prime}\right), \forall \omega \in K(\mathfrak{B}),
$$

and therefore, $U^{*} U, U^{*} U^{\prime}$ are projections. $j(\omega)=\omega$, if and only if $\omega \in H_{A}$ and hence $j \in \mathscr{P}_{F}$ and similarly $j^{\prime} \in \mathscr{P}_{F}$. Clearly, $j j^{\prime}=j^{\prime} j=0$ and $E_{j}+E_{j^{\prime}}$ $=A+A^{\prime}=e$. It follows that $j$ is strongly (a) (and (c))-repeatable with complementary repeatable operation $j^{\prime}$.

Finally, notice that $j \in \mathscr{P}^{\text {II }}$ is always a strong operation when $U \in \mathfrak{B}$ or $\mathfrak{B}^{\prime}$ and in particular, every strongly (a) (or (c))-repeatable operation is a strong operation. The properties of $\mathscr{P}^{\mathrm{II}}$ discussed above are listed below. 
Proposition 5.2. Let $j, j^{\prime} \in \mathscr{P}$ II be defined by (5.4), (5,5) and let $S_{U}, S_{U^{*}}$ be the supports of $U, U^{*}$ respectively, Then,

(i) $Q_{A}=e-S_{U}$ and if $U \in \mathfrak{B}, E_{j}=S_{U^{*}}$.

(ii) $j \in \mathscr{P}_{S}$ if and only if $U$ is unitary.

(iii) $j \in \mathscr{P}_{R}$ with complementary repeatable operation $j^{\prime}$ if and only if $S_{U^{*}} \leqq P_{A}, S_{U^{*}} \leqq P_{e-A}$.

(iv) $j$ is (a) (or (c))-repeatable with complementary repeatable operation $j^{\prime}$ if and only if $U, U^{\prime}$ are partial isometries such that $U^{*} U+U^{\prime *} U^{\prime}=e$, $U U^{*} \leqq U^{*} U, U^{\prime} U^{\prime *} \leqq U^{*} U^{\prime}, E_{j}+E_{j^{\prime}}=e$.

(v) If $U, U^{\prime} \in \mathfrak{B}, j$ is (a) (or (c))-repeatable with complementary repeatable operation $j^{\prime}$ if and only if $U, U^{\prime}$ are partial isometries such that $U^{*} U=U U^{*}, U^{\prime *} U^{\prime}=U^{\prime} U^{*}, U^{*} U+U^{*} U^{\prime}=e$.

(vi) $j$ is strongly (a) (or (c))-repeatable with complementary repeatable operation $j^{\prime}$ if and only if

$$
j(\omega)=A \omega A, j^{\prime}(\omega)=A^{\prime} \omega A^{\prime}, \forall \omega \in K(B)
$$

where $A^{\prime}=e-A$.

(vii) $j$ is a strong operation if $U \in \mathfrak{B}$ or $\mathfrak{B}^{\prime}$.

Clearly, the most important result of Prop. 5.2 is (vi) which characterizes the set of strongly (a) (or (c))-repeatable operations in $\mathscr{P}^{\mathrm{II}}$ as those of the form

$$
j(\omega)=A \omega A, \forall \omega \in K(\mathfrak{B})
$$

where $A$ is a projection in $\mathfrak{B}$. These are the operations suggested by Von Neumann's measurement theory [21].

Acknowledgements. The author wishes to express his gratitude to M. A. Gerzon for allowing him to use his unpublished results on split extremal sets, to E. M. Alfsen for a pre-print of his joint paper with T. B. Anderson and to F. C. Holroyd who, in discussion, made several helpful suggestions. The author also wishes to express his appreciation to the referee for drawing to his attention two papers which deal with related topics. These are: B. Mielnik, Theory of filters, Commun. Math. Phys. 15, $1-46$ (1969) and R. Giles, Foundations of Quantum Mechanics, J. Math. Phys. 11, 2139-2160 (1970).

\section{References}

1. Alfsen, E. M., Anderson, T. B.: Split faces of compact convex sets (pre-print).

2. Cunningham, F.: $L$-structure in $L$-spaces. Trans. Am. Math. Soc. 95, 274-299 (1960).

3. Davies, E. B.: Quantum stochastic processes. Commun. Math. Phys. 15, 277-304 (1969).

4. -, Lewis, J. T.: An operational approach to quantum probability. Commun. Math. Phys. 17, 239-260 (1970).

5. Dixmier, J.: Les $C^{*}$-algèbres et leurs representations. Paris: Gauthier-Villars 1964.

6. - Les algèbres d'operateurs dans l'espace hilbertien. Paris: Gauthier-Villars 1969.

7. Edwards, C. M.: The operational approach to algebraic quantum theory I. Commun. Math. Phys. 16, 207-230 (1970). 
8. Edwards, C. M., Gerzon, M. A.: Monotone convergence in partially ordered vector spaces. Ann. Inst. Henri Poincaré A 12, 323-328 (1970).

9. Effros, E. G.: Order ideals in a $C^{*}$-algebra and its dual. Duke Math. J. 30, 391-412 (1963).

10. Ellis, A. J.: The duality of partially ordered normed vector spaces. J. London Math. Soc. 39, 730-744 (1964).

11. - Linear operators in partially ordered vector spaces. J. London Math. Soc. 41, 323-332 (1966).

12. Gerzon, M. A.: Split faces of convex sets (in preparation).

13. Haag, R., Kastler, D.: An algebraic approach to quantum field theory. J. Math. Phys. 5, 846-861 (1964).

14. Hellwig, K.-E., Kraus, K.: Pure operations and measurements. Commun. Math. Phys. 11, 214-220 (1969).

15. - - Operations and measurements II. Commun. Math. Phys. 16, 142-147 (1970).

16. Kadison, R. V.: Isometries of operator algebras. Ann. Math. 54, 325-338 (1950).

17. Mackey, G. W.: Mathematical foundations of quantum mechanics. New York: Benjamin 1963.

18. Prosser, R. T.: On the ideal structure of operator algebras. Mem. Am. Math. Soc. 45 (1963).

19. Segal, I. E.: Postulates for general quantum mechanics. Ann. Math. 48, 930-948 (1948).

20. Stormer, E.: Positive linear maps of operator algebras. Acta Math. 110, 233-278 (1963).

21. Von Neumann, J.: Mathematical foundations of quantum mechanics. Princeton: Princeton University Press 1957.

C. M. Edwards

The Queen's College

Oxford OX1 4AW, G.B. 